\title{
Multiplicity of positive solutions for Hadamard fractional differential equations with $p$-Laplacian operator
}

\author{
Sabbavarapu Nageswara Rao ${ }^{1 *}$, Manoj Singh'1 and M. Zico Meetei
}

"Correspondence:

snrao@jazanu.edu.sa

1 Department of Mathematics,

Faculty of Science, Jazan University,

Jazan, Saudi Arabia

\section{Springer}

\begin{abstract}
In this paper, we investigate the multiplicity results of some positive solutions for a system of Hadamard fractional differential equations with parameters and $p$-Laplacian operator subject to three-point boundary conditions which contains fractional derivatives. The proofs of our main result, multiplicity of positive solutions, are derived in terms of different values of parameters by using Guo-Krasnosel'skii's fixed point theorem.
\end{abstract}

MSC: 34B09; 34B15; 34B18; 34B27

Keywords: Hadamard fractional equation; Green function; Positive solution; Fixed point theorem; Cone

\section{Introduction}

Fractional calculus has gained considerable attention from both theoretical and applied points of view in recent years. There are numerous applications in a variety of fields such as the signal processing [1], the image processing [2], the control theory [3], the behavior of viscoelastic and visco-plastic materials under external influences $[4,5]$, the bioengineering $[6,7]$, and so on. In addition there are some applications of fractional calculus within various fields of mathematics itself, e.g., in the analytical investigation of various types of special functions [8]. Therefore, the fractional differential equation has been widely focused on and studied in depth. In fact, fractional differential equations have attracted more and more attention for their useful applications in various fields such as economics, science, and engineering, see [9-13] and the references therein.

Turbulent flow in a porous medium is a fundamental mechanics problem. For studying this type of problem, Leibenson [14] introduced differential equations with $p$-Laplacian operator

$$
\left(\phi_{p}\left(u^{\prime}(t)\right)\right)^{\prime}=f(t, u(t)) .
$$

(c) The Author(s) 2020. This article is licensed under a Creative Commons Attribution 4.0 International License, which permits use, sharing, adaptation, distribution and reproduction in any medium or format, as long as you give appropriate credit to the original author(s) and the source, provide a link to the Creative Commons licence, and indicate if changes were made. The images or other third party material in this article are included in the article's Creative Commons licence, unless indicated otherwise in a credit line to the material. If material is not included in the article's Creative Commons licence and your intended use is not permitted by statutory regulation or exceeds the permitted use, you will need to obtain permission directly from the copyright holder. To view a copy of this licence, visit http://creativecommons.org/licenses/by/4.0/. 
The study of differential equation with $p$-Laplacian operator is of significance theoretically and practically. It is quite natural to study fractional differential equation relative to the above equation.

Recently, many scholars have paid more attention to the fractional differential equation boundary value problems and associated with $p$-Laplacian operator, see [15-27].

In [15] Bachar et al. proved the existence and uniqueness and global asymptotic behavior of a positive continuous solution to the following fractional Navier boundary value problem:

$$
\left\{\begin{array}{l}
D^{\alpha}\left(D^{\beta} u\right)(x)+u(x) f(x, u(x))=0, \quad 0<x<1, \\
\lim _{x \rightarrow 0^{+}} D^{\beta-1} u(x)=0, \quad \lim _{x \rightarrow 0^{+}} D^{\alpha-1}\left(D^{\beta} u\right)(x)=\xi \\
u(1)=0, \quad D^{\beta} u(1)=-\zeta,
\end{array}\right.
$$

where $\alpha, \beta \in(1,2], D^{\alpha}$ and $D^{\beta}$ stand for the standard Riemann-Liouville fractional derivative and $\xi, \zeta \geq 0$ are such that $\zeta+\xi>0$.

In [23] Chai investigated the existence and multiplicity of positive solutions for a class of boundary value problems of fractional differential equations with $p$-Laplacian operator:

$$
\left\{\begin{array}{l}
D_{0^{+}}^{\beta}\left(\phi_{p}\left(D_{0^{+}}^{\alpha} u(t)\right)\right)+f(t, u(t))=0, \quad 0<t<1, \\
u(0)=0, \quad u(1)+\sigma D_{0^{+}}^{\gamma} u(1)=0, \quad D_{0^{+}}^{\alpha} u(0)=0,
\end{array}\right.
$$

where $1<\alpha \leq 2,0<\beta \leq 1,0<\gamma \leq 1,0 \leq \alpha-\gamma-1, \sigma$ is a positive constant number, and $D_{0^{+}}^{\alpha}, D_{0^{+}}^{\beta}, D_{0^{+}}^{\gamma}$ are the standard Riemann-Liouville derivatives, by means of the fixed point theorem on cones.

In [25] Tian et al. investigated the existence of positive solutions for a boundary value problem of fractional differential equations with $p$-Laplacian operator

$$
\left\{\begin{array}{l}
D_{0^{+}}^{\beta}\left(\phi_{p}\left(D_{0^{+}}^{\alpha} y(x)\right)\right)=f(x, y(x)), \quad 0<x<1, \\
y(0)=y^{\prime}(0)=y(1)=D_{0^{+}}^{\alpha} y(0)=0, \quad D_{0^{+}}^{\alpha} y(1)=\lambda D_{0^{+}}^{\alpha} y(\xi),
\end{array}\right.
$$

where $\alpha, \beta \in \mathbb{R}, 2<\alpha \leq 3,1<\beta \leq 2$, and $\xi \in(0,1), \lambda \in[0,+\infty), \phi_{p}(z)=|z|^{p-2} z, p>1, D_{0^{+}}^{\alpha}$ is the Riemann-Liouville fractional derivative, and $f \in([e, 1] \times[0,+\infty),[0,+\infty))$. By using Krasnosel'skii's fixed point theorem, we give some multiplicity results.

In [26] Tian et al. considered the boundary value problem of fractional differential equations with $p$-Laplacian operator

$$
\left\{\begin{array}{l}
D^{\gamma}\left(\phi_{p}\left(D^{\alpha} u(t)\right)\right)=f(t, u(t)), \quad 0<t<1, \\
u(0)=D^{\alpha} u(0)=0, \quad D^{\beta} u(1)=a D^{\beta} u(\xi), \quad D^{\alpha} u(1)=b D^{\alpha} u(\eta),
\end{array}\right.
$$

where $\alpha, \beta, \gamma \in \mathbb{R}, 1<\alpha, \gamma \leq 2, \beta>0$ and $1+\beta \leq \alpha \xi, \eta \in(0,1) a, b \in[0, \infty), 1-a \xi^{\alpha-\beta-1}>$ $0,1-b^{p-1} \eta^{\gamma-1}>0 . D^{\alpha}$ is the Riemann-Liouville fractional derivative and $f \in([0,1] \times$ $[0,+\infty),[0,+\infty))$. Some existence results of positive solutions were obtained by using the monotone iterative method.

The system of fractional differential equations boundary value problems with $p$ Laplacian operator has also received much attention and has developed very rapidly, see [28-36]. 
In [32] He and Song discussed the following fractional order differential system with -Laplacian operator:

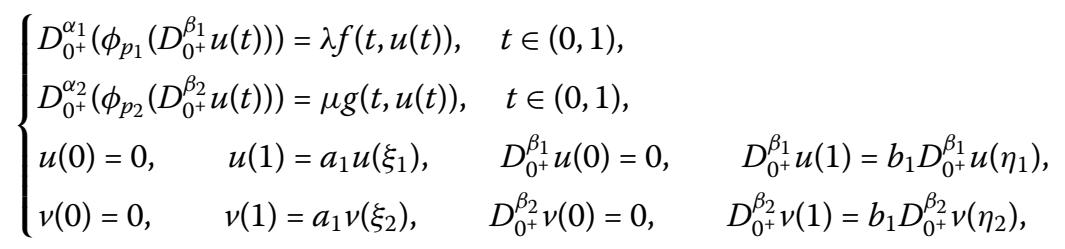

where $\alpha_{i}, \beta_{i} \in(1,2], D_{0^{+}}^{\alpha_{i}}$ and $D_{0^{+}}^{\beta_{i}}$ are the standard Riemann-Liouville derivatives, $\xi_{i}, \eta_{i} \in$ $(0,1), a_{i}, b_{i} \in[0,1], i=1,2, \lambda$ and $\mu$ are positive parameters. The uniqueness of solution was established by using the Banach contraction mapping principle.

In [36] Luca established the existence and nonexistence of positive solutions for a system of nonlinear Riemann-Liouville fractional differential equations with parameters and $p$ Laplacian operator subject to multi-point boundary conditions

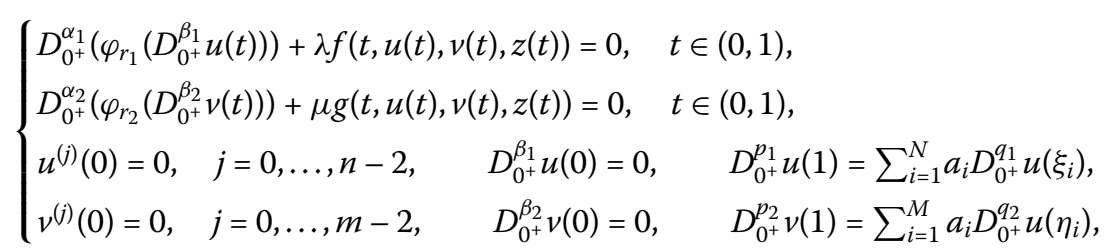

where $\alpha_{1}, \alpha_{2} \in(0,1], \beta_{1} \in(n-1, n], \beta_{2} \in(m-1, m], n, m \in \mathbb{N}, n, m \geq 3, p_{1}, p_{2}, q_{1}, q_{2} \in$ $\mathbb{R}, p_{1} \in[1, n-2], p_{2} \in[1, m-2], q_{1} \in\left[0, q_{1}\right], q_{2} \in\left[0, p_{2}\right], \xi_{i}, a_{i} \in \mathbb{R}$ for all $i=1, \ldots, N(N \in$ $\mathbb{N}) 0<\xi_{1}<\cdots<\xi_{N}<1, \eta_{i}, b_{i} \in \mathbb{R}$ for all $i=1, \ldots, M(M \in \mathbb{N}), 0<\eta_{1}<\cdots<\eta_{M} \leq 1, r_{1}, r_{2}>$ $1, \lambda, \mu>0, f, g \in C([0,1] \times[0, \infty) \times[0, \infty),[0, \infty))$.

It has been noticed that most of the above-mentioned work on the topic is based on Riemann-Liouville or Caputo fractional derivatives. As we know, Hadamard fractional derivative is also a famous fractional derivative given by Hadamard [37] in 1892, and we can find this kind of derivative in the literature. The key of this definition involves a logarithmic function of arbitrary exponent. In the past decades, there were more studies on Hadamard fractional differential equations under different boundary conditions, see [3854].

Huang and Liu [39] established the existence and nonexistence of positive solutions for a class of boundary value problems of nonlinear Hadamard fractional differential equation with a parameter

$$
\left\{\begin{array}{l}
\left({ }^{H} D^{\alpha} x\right)(t)+\lambda a(t) f(x(t))=0, \quad t \in[1, e], \\
x(1)=(\delta x)(1)=(\delta x)(e)=0, \quad \alpha \in(2,3]
\end{array}\right.
$$

where $\lambda$ is a positive parameter, ${ }^{H} D^{\alpha}$ is the left-sided Hadamard fractional derivative of order $\alpha,(\delta x)(t)=t d x(t) / d t, a:(1, e) \rightarrow[0, \infty)$ and $f:[0, \infty) \rightarrow[0, \infty)$ are two continuous functions. 
Yang [41] established positive solutions for the coupled Hadamard fractional integral boundary value problems

$$
\left\{\begin{array}{l}
{ }^{H} D^{\alpha} u(t)+\lambda f(t, u(t), v(t))=0, \quad t \in(1, e), \lambda>0, \\
{ }^{H} D^{\beta} v(t)+\lambda g(t, u(t), v(t))=0, \quad t \in(1, e), \lambda>0, \\
u^{(j)}(1)=v^{(j)}(1)=0, \quad 0 \leq j \leq n-2, \\
u(e)=\mu \int_{1}^{e} v(s) \frac{d s}{s}, \quad v(e)=v \int_{1}^{e} u(s) \frac{d s}{s}
\end{array}\right.
$$

where $\alpha, \beta \in(n-1, n]$ and $n \geq 3,{ }^{H} D^{\alpha},{ }^{H} D^{\beta}$ are the Hadamard fractional derivatives of fractional order $\alpha$ and $\beta$ respectively.

Yang [42] investigated the existence of at least one positive solution for Hadamard fractional differential equations system

$$
\left\{\begin{array}{l}
{ }^{H} D^{\alpha} u(t)+\lambda f(t, u(t), v(t))=0, \quad t \in(1, e), \\
{ }^{H} D^{\beta} v(t)+\lambda g(t, u(t), v(t))=0, \quad t \in(1, e), \\
u^{(j)}(1)=v^{(j)}(1)=0, \quad 0 \leq j \leq n-2, \\
u(e)=a v(\xi), \quad v(e)=b u(\eta), \quad \xi, \eta \in(1, e),
\end{array}\right.
$$

where $\lambda, a, b$ are three parameters, $\alpha, \beta \in(n-1, n]$ are two real numbers, and $n \geq 3$, by applying Guo-Krasnoselskii's fixed point theorem. Zhang and Liu [47] investigated the existence of solutions for several higher order integral boundary value problems of Hadamardtype fractional differential equations on an infinite interval by using the monotone iterative technique and Mawhin's continuation theorem. In [48], Ahmad and Ntouyas discussed the following coupled Hadamard-type FDEs with Hadamard-type integral boundary conditions:

$$
\left\{\begin{array}{l}
{ }^{H} D^{\alpha} u(t)=f(t, u(t), v(t)), \quad 1<\alpha \leq 2,1<t<e, \\
{ }^{H} D^{\beta} v(t)=g(t, u(t), v(t)), \quad 1<\beta \leq 2,1<t<e, \\
u(1)=0, \quad u(e)=\frac{1}{\Gamma(\gamma)} \int_{1}^{\sigma_{1}}\left(\ln \frac{\sigma_{1}}{s}\right)^{\gamma-1} \frac{u(s)}{s} d s, \\
v(1)=0, \quad v(e)=\frac{1}{\Gamma(\gamma)} \int_{1}^{\sigma_{2}}\left(\ln \frac{\sigma_{2}}{s}\right)^{\gamma-1} \frac{v(s)}{s} d s,
\end{array}\right.
$$

where $\gamma>0,1<\sigma_{1}<e, 1<\sigma_{2}<e,{ }^{H} D^{(\cdot)}$ is the Hadamard-type fractional derivative of fractional order. By using Leray-Schauder's alternative and Banach's contraction principle, the authors obtained the existence and uniqueness of solutions, respectively.

Recentely, Rodica Luca [22] studied the existence and nonexistence of positive solutions for a system with three nonlinear Riemann-Liouville fractional differential equations with multi-point boundary conditions which contain fractional derivatives by using Guo-Krasnosel'skii's fixed point theorem, and Alesemi [54] investigated eigenvalue intervals for a system with three nonlinear Hadamard fractional differential equations with $p$-Laplacian operator by using Guo-Krasnosel'skii's fixed point theorem on cones. Various existence results for positive solutions have been derived in terms of different values of parameters.

Motivated by the aforementioned work, we investigate in this paper the existence of multiple positive solutions for the following Hadamard fractional differential equations 
with $p_{1}$-Laplacian, $p_{2}$-Laplacian, and $p_{3}$-Laplacian operators:

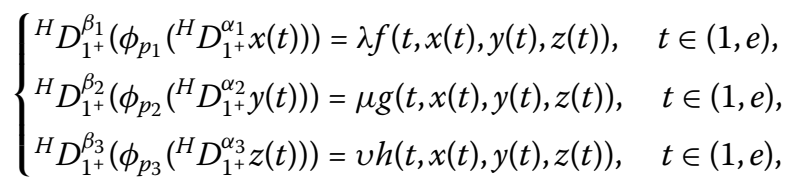

subject to the boundary conditions

$$
\left\{\begin{array}{l}
x(1)=x^{\prime}(1)=0, \quad \xi_{1} x(e)+\eta_{1}^{H} D_{1^{+}}^{\gamma_{1}} x(e)=0, \\
\phi_{p_{1}}\left({ }^{H} D_{1^{+}}^{\alpha_{1}} x(1)\right)=0, \quad \phi_{p_{1}}\left({ }^{H} D_{1^{+}}^{\alpha_{1}} x(e)\right)=\vartheta_{1} \phi_{p_{1}}\left({ }^{H} D_{1^{+}}^{\alpha_{1}} x(\delta)\right), \\
y(1)=y^{\prime}(1)=0, \quad \xi_{2} y(e)+\eta_{2}^{H} D_{1^{+}}^{\gamma_{2}} y(e)=0, \\
\phi_{p_{2}}\left({ }^{H} D_{1^{+}}^{\alpha_{2}} y(1)\right)=0, \quad \phi_{p_{2}}\left({ }^{H} D_{1^{+}}^{\alpha_{2}} y(e)\right)=\vartheta_{2} \phi_{p_{2}}\left({ }^{H} D_{1^{+}}^{\alpha_{2}} y(\delta)\right), \\
z(1)=z^{\prime}(1)=0, \quad \xi_{3} z(e)+\eta_{3}^{H} D_{1^{+}}^{\gamma_{3}} z(e)=0, \\
\phi_{p_{3}}\left({ }^{H} D_{1^{+}}^{\alpha_{3}} z(1)\right)=0, \quad \phi_{p_{3}}\left({ }^{H} D_{1^{+}}^{\alpha_{3}} z(e)\right)=\vartheta_{3} \phi_{p_{3}}\left({ }^{H} D_{1^{+}}^{\alpha_{3}} z(\delta)\right),
\end{array}\right.
$$

where $\phi_{p_{i}}(s)=|s|^{p_{i}-2} s, p_{i}>1, \phi_{p_{i}}^{-1}=\phi_{q_{i}}, \frac{1}{p_{i}}+\frac{1}{q_{i}}=1, i=1,2,3, f, g, h \in C\left([1, e] \times[0,+\infty)^{3}\right.$, $[0,+\infty)), \lambda, \mu, v$ are positive parameters, $\xi_{i}, \eta_{i}, \vartheta_{i} \in[0, \infty), \delta \in(1, e), \alpha_{i} \in(2,3], \beta_{i}, \gamma_{i} \in$ $(1,2], i=1,2,3$, and ${ }^{H} D_{1^{+}}^{k}$ denotes the Hadamard fractional order $k$ (for $k=\alpha_{i}, \beta_{i}, \gamma_{i}, i=$ $1,2,3)$.

Under some assumptions on $f, g$, and $h$, we give intervals for the parameters $\lambda, \mu$, and $v$ such that positive solutions of (1)-(2) exist. By a positive solution of problem (1)-(2), we mean a triplet of functions $(x, y, z) \in(C([1, e],[0, \infty)))^{3}$ satisfying (1)-(2) with $x(t)>0$ for all $t \in[1, e]$, or $y(t)>0$ for all $t \in[1, e]$, or $z(t)>0$ for all $t \in[1, e]$ and $(x, y, z) \neq(0,0,0)$.

We use the following notations for our convenience:

$$
\begin{aligned}
& \sigma_{i}=\int_{1}^{e} G_{i}(e, s) \phi_{q_{i}}\left(\int_{1}^{e} l_{i}(\tau) \kappa_{i}(\tau) \frac{d \tau}{\tau}\right) \frac{d s}{s}, \quad i=1,2,3 ; \\
& \rho_{j}=\int_{s \in I} G_{j}(e, s) \phi_{q_{j}}\left(\int_{s \in I} \varsigma(\tau) K_{j 1}(\tau, \tau) \frac{d \tau}{\tau}\right) \frac{d s}{s}, \quad j=1,2,3 .
\end{aligned}
$$

We make the following assumptions throughout:

(A1) The functions $f, g, h:[1, e] \times[0,+\infty)^{3} \rightarrow[0,+\infty)$ are continuous.

(A2) $\xi_{i}, \eta_{i}, \vartheta_{i}>0,2<\alpha_{i} \leq 3,1<\beta_{i}, \gamma_{i} \leq 2, \eta_{i}\left(\gamma_{i}-1\right)>\frac{\xi_{i} \Gamma\left(\alpha_{i}-\gamma_{i}\right)}{\Gamma\left(\alpha_{i}\right)}, 1-\vartheta_{i}(\ln \delta)^{\beta_{i}-1}>0$, and $\Delta_{i}=\eta_{i} \Gamma\left(\alpha_{i}\right)+\xi_{i} \Gamma\left(\alpha_{i}-\gamma_{i}\right)>0, i=1,2,3$.

(A3) The functions $f(t, x, y, z) \leq \kappa_{1}(t) u_{1}(t, x, y, z), g(t, x, y, z) \leq \kappa_{2}(t) u_{2}(t, x, y, z)$, $h(t, x, y, z) \leq \kappa_{3}(t) u_{3}(t, x, y, z),(t, x, y, z) \in[1, e] \times[0, \infty) \times[0, \infty), \times[0, \infty)$, where $u_{i} \in C[[1, e] \times[0, \infty) \times[0, \infty) \times[0, \infty),[0, \infty)]$, and $\kappa_{i} \in C[[1, e],[0, \infty)]$ satisfy $\int_{1}^{e} \kappa_{i}(s) \frac{d s}{s}<\infty, i=1,2,3$.

(A4) For $I=\left[e^{1 / 4}, e^{3 / 4}\right] \subset[1, e]$, we introduce the following extreme limits:

$$
\begin{array}{rlrl}
f_{0} & =\lim _{x+y+z \rightarrow 0} \min _{t \in I} \frac{f(t, x, y, z)}{(x+y+z)^{p_{1}-1}}, & f_{\infty}=\lim _{\bar{x}+y+z \rightarrow \infty} \min _{t \in I} \frac{f(t, x, y, z)}{(x+y+z)^{p_{1}-1}}, \\
g_{0}=\frac{\lim }{x+y+z \rightarrow 0} \min _{t \in I} \frac{g(t, x, y, z)}{(x+y+z)^{p_{2}-1}}, & g_{\infty}=\lim _{\frac{1}{x+y+z \rightarrow \infty}} \min _{t \in I} \frac{g(t, x, y, z)}{(x+y+z)^{p_{2}-1}}, \\
h_{0}=\frac{\lim }{x+y+z \rightarrow 0} \min _{t \in I} \frac{h(t, x, y, z)}{(x+y+z)^{p_{3}-1}}, & h_{\infty}=\frac{\lim }{x+y+z \rightarrow \infty} \min _{t \in I} \frac{h(t, x, y, z)}{(x+y+z)^{p_{3}-1}},
\end{array}
$$




$$
u_{i 0}=\lim _{x+y+z \rightarrow 0} \max _{t \in[1, e]} \frac{u_{i}(t, x, y, z)}{(x+y+z)^{p_{i}-1}}, \quad u_{i \infty}=\lim _{x+y+z \rightarrow \infty} \max _{t \in[1, e]} \frac{u_{i}(t, x, y, z)}{(x+y+z)^{p_{i}-1}} .
$$

In the definition of the extreme limits above, the variables $x, y$, and $z$ are nonnegative with $f_{0}, f_{\infty}, g_{0}, g_{\infty}, h_{0}, h_{\infty}, u_{i 0}, u_{i \infty} \in[0, \infty), i=1,2,3$.

The aim of this paper is to establish some existence and multiplicity results of positive solutions for system (1)-(2) in explicit intervals for $\lambda, \mu$, and $\nu$. The rest of the paper is organized as follows: In Sect. 2, we give some properties of the Green's function which are needed later. Also, we state Guo-Krasnosel'skii's fixed point theorem on cones, and we prove a key lemma used in the proofs of our main results. In Sect. 3, we discuss the existence of multiplicity results of positive solutions of system (1)-(2). The intervals in which the parameters $\lambda, \mu$, and $v$ can guarantee the existence of a solution are obtained. At the end, we give an example to illustrate our main results.

\section{Preliminaries and lemmas}

We present here the definitions, some lemmas from the theory of Hadamard fractional calculus, and some auxiliary results that will be used to prove our main theorems.

Definition 2.1 ([9]) The left-sided Hadamard fractional integrals of order $\alpha \in \mathbb{R}^{+}$of the function $h(t)$ are defined by

$$
\left({ }^{H} I^{\alpha} h\right)(t)=\frac{1}{\Gamma(\alpha)} \int_{1}^{t}\left(\ln \frac{t}{s}\right)^{\alpha-1} h(s) \frac{d s}{s} \quad(1 \leq t \leq e),
$$

where $\Gamma(\cdot)$ is the gamma function.

Definition 2.2 ([9]) The left-sided Hadamard fractional derivatives of order $\alpha \in(n-$ $1, n], n \in Z^{+}$of the function $h(t)$ are defined by

$$
\left({ }^{H} D^{\alpha} h\right)(t)=\frac{1}{\Gamma(n-\alpha)}\left(t \frac{d}{d t}\right)^{n} \int_{1}^{t}\left(\ln \frac{t}{s}\right)^{n-\alpha+1} h(s) \frac{d s}{s} \quad(1 \leq t \leq e),
$$

where $\Gamma(\cdot)$ is the gamma function.

Lemma 2.1 ([9]) If $a, \alpha, \beta>0$, then

$$
\left(D_{a}^{\alpha}\left(\ln \frac{t}{a}\right)^{\beta-1}\right)(x)=\frac{\Gamma(\beta)}{\Gamma(\beta-\alpha)}\left(\ln \frac{x}{a}\right)^{\beta-\alpha-1} .
$$

Lemma 2.2 ([9]) Let $q>0$ and $u \in C[1, \infty) \cap L^{1}[1, \infty)$. Then the Hadamard fractional differential equation ${ }^{H} D^{q} u(t)=0$ has the solution

$$
u(t)=\sum_{i=1}^{n} c_{i}(\ln t)^{q-1}
$$

and the following formula holds:

$$
{ }^{H} I^{q H} D^{q} u(t)=u(t)+\sum_{i=1}^{n} c_{i}(\ln t)^{q-i}
$$

where $c_{i} \in R, i=1,2, \ldots, n$, and $n-1<q<n$. 
Lemma 2.3 Let $\Delta_{1}=\eta_{1} \Gamma\left(\alpha_{1}\right)+\xi_{1} \Gamma\left(\alpha_{1}-\gamma_{1}\right)>0, \omega \in C[1, e]$, and $2<\alpha_{1} \leq 3$. Then the unique solution of

$$
\left\{\begin{array}{l}
{ }^{H} D_{1^{+}}^{\alpha_{1}} x(t)+\omega(t)=0, \quad 1<t<e, \\
x(1)=x^{\prime}(1)=0, \quad \xi_{1} x(e)+\eta_{1}^{H} D_{1^{+}}^{\gamma_{1}} x(e)=0
\end{array}\right.
$$

is $x(t)=\int_{1}^{e} G_{1}(t, s) \omega(s) \frac{d s}{s}$, where

$$
\begin{aligned}
& G_{1}(t, s)= \begin{cases}G_{11}(t, s), & 1 \leq t \leq s \leq e \\
G_{12}(t, s), & 1 \leq s \leq t \leq e\end{cases} \\
& G_{11}(t, s)=\frac{1}{\Delta_{1}}\left[\eta_{1}(1-\ln s)^{-\gamma_{1}}+\frac{\xi_{1} \Gamma\left(\alpha_{1}-\gamma_{1}\right)}{\Gamma\left(\alpha_{1}\right)}\right](\ln t)^{\alpha_{1}-1}(1-\ln s)^{\alpha_{1}-1}, \\
& G_{12}(t, s)=\frac{1}{\Delta_{1}}\left[\eta_{1}(1-\ln s)^{-\gamma_{1}}+\frac{\xi_{1} \Gamma\left(\alpha_{1}-\gamma_{1}\right)}{\Gamma\left(\alpha_{1}\right)}\right](\ln t)^{\alpha_{1}-1}(1-\ln s)^{\alpha_{1}-1} \\
& -\frac{1}{\Gamma\left(\alpha_{1}\right)}\left(\ln \frac{t}{s}\right)^{\alpha_{1}-1}
\end{aligned}
$$

Proof Assume that $x \in C^{\left[\alpha_{1}\right]+1}[1, e]$ is a solution of Hadamard fractional order BVPs (3) and is uniquely expressed as

$$
{ }^{H} I_{1^{+}}^{\alpha_{1} H} D_{1^{+}}^{\alpha_{1}} x(t)=-{ }^{H} I_{1^{+}}^{\alpha_{1}} \omega(t)
$$

such that

$$
x(t)=c_{1}(\ln t)^{\alpha_{1}-1}+c_{2}(\ln t)^{\alpha_{1}-2}+c_{3}(\ln t)^{\alpha_{1}-3}-\frac{1}{\Gamma\left(\alpha_{1}\right)} \int_{1}^{t}\left(\ln \frac{t}{s}\right)^{\alpha_{1}-1} \omega(s) \frac{d s}{s}
$$

for some $c_{i} \in \mathbb{R}, i=1,2,3$. From the boundary condition $x(1)=x^{\prime}(1)=0$, we have $c_{2}=c_{3}=$ 0 . Hence $x(t)=c_{1}(\ln t)^{\alpha_{1}-1}-\frac{1}{\Gamma\left(\alpha_{1}\right)} \int_{1}^{t}\left(\ln \frac{t}{s}\right)^{\alpha_{1}-1} \omega(s) \frac{d s}{s}$ and

$$
{ }^{H} D_{1^{+}}^{\gamma_{1}}(x(t))=c_{1} \frac{\Gamma\left(\alpha_{1}\right)}{\Gamma\left(\alpha_{1}-\gamma_{1}\right)}(\ln t)^{\alpha_{1}-1}-\frac{1}{\Gamma\left(\alpha_{1}-\gamma_{1}\right)} \int_{1}^{t}\left(\ln \frac{t}{s}\right)^{\alpha_{1}-\gamma_{1}-1} \omega(s) \frac{d s}{s} .
$$

Consequently, we obtain from the boundary condition $\xi_{1} x(e)+\eta_{1}^{H} D_{1^{+}}^{\gamma_{1}} x(e)=0$, we have

$$
c_{1}=\frac{1}{\Delta_{1}} \int_{1}^{e}\left[\eta_{1}(1-\ln s)^{-\gamma_{1}}+\frac{\xi_{1} \Gamma\left(\alpha_{1}-\gamma_{1}\right)}{\Gamma\left(\alpha_{1}\right)}\right](1-\ln s)^{\alpha_{1}-1} \omega(s) \frac{d s}{s} .
$$

As a result,

$$
\begin{aligned}
u(t)= & \frac{1}{\Delta_{1}} \int_{1}^{e}\left[\eta_{1}(1-\ln s)^{-\gamma_{1}}+\frac{\xi_{1} \Gamma\left(\alpha_{1}-\gamma_{1}\right)}{\Gamma\left(\alpha_{1}\right)}\right](1-\ln s)^{\alpha_{1}-1}(\ln t)^{\alpha_{1}-1} \omega(s) \frac{d s}{s} \\
& -\frac{1}{\Gamma\left(\alpha_{1}\right)} \int_{1}^{t}\left(\ln \frac{t}{s}\right)^{\alpha_{1}-1} \omega(s) \frac{d s}{s} \\
= & \int_{1}^{t}\left[\frac{1}{\Delta_{1}}\left[\eta_{1}(1-\ln s)^{-\gamma_{1}}+\frac{\xi_{1} \Gamma\left(\alpha_{1}-\gamma_{1}\right)}{\Gamma\left(\alpha_{1}\right)}\right](\ln t)^{\alpha_{1}-1}(1-\ln s)^{\alpha_{1}-1}\right.
\end{aligned}
$$




$$
\begin{aligned}
& \left.-\frac{1}{\Gamma\left(\alpha_{1}\right)}\left(\ln \frac{t}{s}\right)^{\alpha_{1}-1}\right] \omega(s) \frac{d s}{s} \\
& +\int_{t}^{e} \frac{1}{\Delta_{1}}\left[\eta_{1}(1-\ln s)^{-\gamma_{1}}+\frac{\xi_{1} \Gamma\left(\alpha_{1}-\gamma_{1}\right)}{\Gamma\left(\alpha_{1}\right)}\right](\ln t)^{\alpha_{1}-1}(1-\ln s)^{\alpha_{1}-1} \omega(s) \frac{d s}{s} \\
= & \int_{1}^{e} G_{1}(t, s) \omega(s) \frac{d s}{s} .
\end{aligned}
$$

Lemma 2.4 Let $2<\alpha_{1} \leq 3,1<\beta_{1} \leq 2$, and $\varphi \in C[1, e]$. Then the unique solution of

$$
\left\{\begin{array}{l}
\left.{ }^{H} D_{1^{+}}{ }^{\beta_{1}}\left(\phi_{p_{1}}{ }^{H} D_{1^{+}}^{\alpha_{1}} x(t)\right)\right)=\varphi(t), \quad 1<t<e, \\
x(1)=x^{\prime}(1)=0 ; \quad \xi_{1} x(e)+\eta_{1}^{H} D_{1^{+} x}^{\gamma_{1}} x(e)=0, \\
\phi_{p_{1}}\left({ }^{H} D_{1^{+}}^{\alpha_{1}} x(1)\right)=0, \quad \phi_{p_{1}}\left({ }^{H} D_{1^{+}}^{\alpha_{1}} x(e)\right)=\vartheta_{1} \phi_{p_{1}}\left({ }^{H} D_{1^{+}}^{\alpha_{1}} x(\delta)\right)
\end{array}\right.
$$

is $x(t)=\int_{1}^{e} G_{1}(t, s) \phi_{q_{1}}\left(\int_{1}^{e} K_{1}(s, \tau) \varphi(\tau) \frac{d \tau}{\tau}\right) \frac{d s}{s}$, where $G_{1}(t, s)$ is defined as (4) and

$$
\begin{aligned}
& K_{1}(t, s)=K_{11}(t, s)+\frac{\vartheta_{1}(\ln t)^{\beta_{1}-1}}{1-\vartheta_{1}(\ln \delta)^{\beta_{1}-1}} K_{11}(\delta, s), \\
& K_{11}(t, s)=\frac{1}{\Gamma\left(\beta_{1}\right)} \begin{cases}(\ln t)^{\beta_{1}-1}(1-\ln s)^{\beta_{1}-1}, & 1 \leq t \leq s \leq e, \\
(\ln t)^{\beta_{1}-1}(1-\ln s)^{\beta_{1}-1}-\left(\ln \frac{t}{s}\right)^{\beta_{1}-1}, & 1 \leq s \leq t \leq e .\end{cases}
\end{aligned}
$$

Proof It follows from Lemma 2.2 and $1<\beta_{1} \leq 2$. An equivalent integral equation for (5) is given by

$$
\phi_{p_{1}}\left({ }^{H} D_{1^{+}}^{\alpha_{1}} x(t)\right)=\frac{1}{\Gamma\left(\beta_{1}\right)} \int_{1}^{t}\left(\ln \frac{t}{\tau}\right)^{\beta_{1}-1} \varphi(\tau) \frac{d \tau}{\tau}+c_{1}(\ln t)^{\beta_{1}-1}+c_{2}(\ln t)^{\beta_{1}-2} .
$$

Note that $\phi_{p_{1}}\left({ }^{H} D_{1^{+}}^{\alpha_{1}} x(1)\right)=0$, we have $c_{2}=0$. Hence,

$$
\begin{aligned}
& \left.\phi_{p_{1}}\left({ }^{H} D_{1^{+}}^{\alpha_{1}} x(e)\right)\right)=\frac{1}{\Gamma\left(\beta_{1}\right)} \int_{1}^{e}(1-\ln \tau)^{\beta_{1}-1} \varphi(\tau) \frac{d \tau}{\tau}+c_{1} \text { and } \\
& \left.\phi_{p_{1}}\left({ }^{H} D_{1^{+}}^{\alpha_{1}} x(\delta)\right)\right)=\frac{1}{\Gamma\left(\beta_{1}\right)} \int_{1}^{\delta}\left(\ln \frac{\delta}{\tau}\right)^{\beta_{1}-1} \varphi(\tau) \frac{d \tau}{\tau}+c_{1}(\ln \delta)^{\beta_{1}-1} .
\end{aligned}
$$

Consequently, $\phi_{p_{1}}\left({ }^{H} D_{1^{+}}^{\alpha_{1}} x(e)\right)=\vartheta_{1} \phi_{p_{1}}\left({ }^{H} D_{1^{+}}^{\alpha_{1}} x(\delta)\right)$ implies that

$$
c_{1}=\frac{1}{1-\vartheta_{1}(\ln \delta)^{\beta_{1}-1}}\left[\int_{1}^{\delta} \frac{\vartheta_{1}\left(\ln \frac{\delta}{\tau}\right)^{\beta_{1}-1}}{\Gamma\left(\beta_{1}\right)} \varphi(\tau) \frac{d \tau}{\tau}-\int_{1}^{e} \frac{(1-\ln \tau)^{\beta_{1}-1}}{\Gamma\left(\beta_{1}\right)} \varphi(\tau) \frac{d \tau}{\tau}\right] .
$$

Therefore,

$$
\begin{aligned}
\phi_{p_{1}}\left({ }^{H} D_{1^{+}+}^{\alpha_{1}} x(t)\right)= & \frac{1}{\Gamma\left(\beta_{1}\right)} \int_{1}^{t}\left(\ln \frac{t}{\tau}\right)^{\beta_{1}-1} \varphi(\tau) \frac{d \tau}{\tau} \\
& +\frac{\vartheta_{1}(\ln t)^{\beta_{1}-1}}{1-\vartheta_{1}(\ln \delta)^{\beta_{1}-1}} \int_{1}^{\delta} \frac{\left(\ln \frac{\delta}{\tau}\right)^{\beta_{1}-1}}{\Gamma\left(\beta_{1}\right)} \varphi(\tau) \frac{d \tau}{\tau} \\
& -\frac{(\ln t)^{\beta_{1}-1}}{1-\vartheta_{1}(\ln \delta)^{\beta_{1}-1}} \int_{1}^{e} \frac{(1-\ln \tau)^{\beta_{1}-1}}{\Gamma\left(\beta_{1}\right)} \varphi(\tau) \frac{d \tau}{\tau}
\end{aligned}
$$




$$
\begin{aligned}
= & \frac{1}{\Gamma\left(\beta_{1}\right)} \int_{1}^{t}\left(\ln \frac{t}{\tau}\right)^{\beta_{1}-1} \varphi(\tau) \frac{d \tau}{\tau} \\
& -\frac{(\ln t)^{\beta_{1}-1}}{\Gamma\left(\beta_{1}\right)} \int_{1}^{e}(1-\ln \tau)^{\beta_{1}-1} \varphi(\tau) \frac{d \tau}{\tau} \\
& +\frac{\vartheta_{1}(\ln t)^{\beta_{1}-1}}{1-\vartheta_{1}(\ln \delta)^{\beta_{1}-1}} \int_{1}^{\delta} \frac{\left(\ln \frac{\delta}{\tau}\right)^{\beta_{1}-1}}{\Gamma\left(\beta_{1}\right)} \varphi(\tau) \frac{d \tau}{\tau} \\
& -\frac{\vartheta_{1}(\ln t)^{\beta_{1}-1}}{1-\vartheta_{1}(\ln \delta)^{\beta_{1}-1}} \int_{1}^{e} \frac{(\ln \delta)^{\beta_{1}-1}(1-\ln \tau)^{\beta_{1}-1}}{\Gamma\left(\beta_{1}\right)} \varphi(\tau) \frac{d \tau}{\tau} \\
= & -\int_{1}^{e} K_{1}(t, \tau) \varphi(\tau) \frac{d \tau}{\tau} .
\end{aligned}
$$

Then the Hadamard fractional order BVP (5) is equivalent to the following problem:

$$
\begin{aligned}
& { }^{H} D_{1^{+}}^{\alpha_{1}} x(t)+\phi_{q_{1}}\left(\int_{1}^{e} K_{1}(t, \tau) \varphi(\tau) \frac{d \tau}{\tau}\right)=0 \quad \text { for } t \in(1, e), \\
& x(1)=x^{\prime}(1)=0 ; \quad \xi_{1} x(e)+\eta_{1}^{H} D_{1^{+}}^{\gamma_{1}} x(e)=0 .
\end{aligned}
$$

In view of Lemma 2.3, we get

$$
x(t)=\int_{1}^{e} G_{1}(t, s) \phi_{q_{1}}\left(\int_{1}^{e} K_{1}(s, \tau) \varphi(\tau) \frac{d \tau}{\tau}\right) \frac{d s}{s} .
$$

Lemma 2.5 Assume that (A2) is satisfied. Then the Green's function $G_{1}(t, s)$ given by (4) satisfies the following inequalities:

(i) $G_{1}(t, s) \geq 0$ for all $(t, s) \in[1, e] \times[1, e]$;

(ii) $G_{1}(t, s) \leq G_{1}(e, s)$ for all $(t, s) \in[1, e] \times[1, e]$;

(iii) $G_{1}(t, s) \geq\left(\frac{1}{4}\right)^{\alpha_{1}-1} G_{1}(e, s)$ for all $(t, s) \in I \times(1, e)$, where $I=\left[e^{1 / 4}, e^{3 / 4}\right]$.

Proof Consider the Green's function $G_{11}(t, s)$ given by (4).

(i) For $1 \leq t \leq s \leq e$.

$$
G_{11}(t, s)=\frac{1}{\Delta_{1}}\left[\eta_{1}(1-\ln s)^{-\gamma_{1}}+\frac{\xi_{1} \Gamma\left(\alpha_{1}-\gamma_{1}\right)}{\Gamma\left(\alpha_{1}\right)}\right](\ln t)^{\alpha_{1}-1}(1-\ln s)^{\alpha_{1}-1} \geq 0 .
$$

Let $1 \leq s \leq t \leq e$. Then

$$
\begin{aligned}
G_{12}(t, s)= & \frac{1}{\Delta_{1}}\left[\eta_{1}(1-\ln s)^{-\gamma_{1}}+\frac{\xi_{1} \Gamma\left(\alpha_{1}-\gamma_{1}\right)}{\Gamma\left(\alpha_{1}\right)}\right](\ln t)^{\alpha_{1}-1}(1-\ln s)^{\alpha_{1}-1} \\
& -\frac{1}{\Gamma\left(\alpha_{1}\right)}\left(\ln \frac{t}{s}\right)^{\alpha_{1}-1} \\
= & \frac{1}{\Delta_{1}}\left[\eta_{1}(1-\ln s)^{-\gamma_{1}}+\frac{\xi_{1} \Gamma\left(\alpha_{1}-\gamma_{1}\right)}{\Gamma\left(\alpha_{1}\right)}\right](\ln t)^{\alpha_{1}-1}(1-\ln s)^{\alpha_{1}-1} \\
& -\frac{1}{\Delta_{1}}\left[\eta_{1}+\frac{\xi_{1} \Gamma\left(\alpha_{1}-\gamma_{1}\right)}{\Gamma\left(\alpha_{1}\right)}\right]\left(1-\frac{\ln s}{\ln t}\right)^{\alpha_{1}-1}(\ln t)^{\alpha_{1}-1} \\
\geq & \frac{1}{\Delta_{1}}\left[\eta_{1}(1-\ln s)^{-\gamma_{1}}+\frac{\xi_{1} \Gamma\left(\alpha_{1}-\gamma_{1}\right)}{\Gamma\left(\alpha_{1}\right)}\right](\ln t)^{\alpha_{1}-1}(1-\ln s)^{\alpha_{1}-1} \\
& -\frac{1}{\Delta_{1}}\left[\eta_{1}+\frac{\xi_{1} \Gamma\left(\alpha_{1}-\gamma_{1}\right)}{\Gamma\left(\alpha_{1}\right)}\right](\ln t)^{\alpha_{1}-1}(1-\ln s)^{\alpha_{1}-1}
\end{aligned}
$$




$$
\geq \frac{\eta_{1}}{\Delta_{1}}\left[(1-\ln s)^{-\gamma_{1}}-1\right](\ln t)^{\alpha_{1}-1}(1-\ln s)^{\alpha_{1}-1} \geq 0,
$$

which implies $G_{1}(t, s) \geq 0$. Hence inequality (i) is proved.

(ii) For $1 \leq t \leq s \leq e$.

$$
\begin{aligned}
\frac{d G_{11}(t, s)}{d t} & =\frac{1}{\Delta_{1}}\left[\eta_{1}(1-\ln s)^{-\gamma_{1}}+\frac{\xi_{1} \Gamma\left(\alpha_{1}-\gamma_{1}\right)}{\Gamma\left(\alpha_{1}\right)}\right]\left(\alpha_{1}-1\right)(\ln t)^{\alpha_{1}-2}(1-\ln s)^{\alpha_{1}-1} \\
& \geq 0
\end{aligned}
$$

Therefore, $G_{1}(t, s)$ is increasing with respect to $t$, which implies that $G_{11}(t, s) \leq G_{11}(e, s)$.

Now for $1 \leq s \leq t \leq e$. Then

$$
\begin{aligned}
\frac{d G_{12}(t, s)}{d t} & \frac{1}{\Delta_{1}}\left[\eta_{1}(1-\ln s)^{-\gamma_{1}}+\frac{\xi_{1} \Gamma\left(\alpha_{1}-\gamma_{1}\right)}{\Gamma\left(\alpha_{1}\right)}\right]\left(\alpha_{1}-1\right)(\ln t)^{\alpha_{1}-2}(1-\ln s)^{\alpha_{1}-1} \\
& -\frac{1}{\Gamma\left(\alpha_{1}\right)}\left(\alpha_{1}-1\right)\left(\ln \frac{t}{s}\right)^{\alpha_{1}-2} \\
= & \frac{1}{\Delta_{1}}\left[\eta_{1}(1-\ln s)^{-\gamma_{1}}+\frac{\xi_{1} \Gamma\left(\alpha_{1}-\gamma_{1}\right)}{\Gamma\left(\alpha_{1}\right)}\right]\left(\alpha_{1}-1\right)(\ln t)^{\alpha_{1}-2}(1-\ln s)^{\alpha_{1}-1} \\
& -\frac{1}{\Delta_{1}}\left[\eta_{1}+\frac{\xi_{1} \Gamma\left(\alpha_{1}-\gamma_{1}\right)}{\Gamma(\alpha)}\right]\left(\alpha_{1}-1\right)\left(1-\frac{\ln s}{\ln t}\right)^{\alpha_{1}-2}(\ln t)^{\alpha_{1}-2} \\
\geq & \frac{\left(\alpha_{1}-1\right)(\ln t)^{\alpha_{1}-2}}{\Delta_{1}}\left[\eta_{1}(1-\ln s)^{-\gamma_{1}}+\frac{\xi_{1} \Gamma\left(\alpha_{1}-\gamma_{1}\right)}{\Gamma\left(\alpha_{1}\right)}\right](1-\ln s)^{\alpha_{1}-1} \\
& -\frac{1}{\Delta_{1}}\left[\eta_{1}+\frac{\xi_{1} \Gamma\left(\alpha_{1}-\gamma_{1}\right)}{\Gamma\left(\alpha_{1}\right)}\right](1-\ln s)^{\alpha_{1}-2} \\
= & \frac{\left(\alpha_{1}-1\right)(\ln t)^{\alpha_{1}-2}}{\Delta_{1}}\left[\eta_{1}(1-\ln s)^{-\left(\gamma_{1}-1\right)}-\eta_{1}-\frac{\xi_{1} \Gamma\left(\alpha_{1}-\gamma_{1}\right)}{\Gamma\left(\alpha_{1}\right)} \ln s\right](1-\ln s)^{\alpha_{1}-2} \\
= & \frac{\left(\alpha_{1}-1\right)(\ln t)^{\alpha_{1}-2}}{\Delta_{1}}\left[\eta_{1}\left(\left(\gamma_{1}-1\right)(\ln s)+\frac{\left(\gamma_{1}-1\right)\left(\gamma_{2}-1\right)}{2}(\ln s)^{2}+\cdots\right)\right. \\
& \left.-\frac{\xi_{1} \Gamma\left(\alpha_{1}-\gamma_{1}\right)}{\Gamma\left(\alpha_{1}\right)}(\ln s)\right](1-\ln s)^{\alpha_{1}-2} \\
= & \frac{\left(\alpha_{1}-1\right)(\ln t)^{\alpha_{1}-2}}{\Delta_{1}}\left[\left(\eta_{1}\left(\gamma_{1}-1\right)-\frac{\xi_{1} \Gamma\left(\alpha_{1}-\gamma_{1}\right)}{\Gamma\left(\alpha_{1}\right)}\right)(\ln s)+O(\ln s)^{2}\right] \\
& \times(1-\ln s)^{\alpha_{1}-2} \\
\geq & 0 .
\end{aligned}
$$

Therefore, $G_{12}(t, s)$ is increasing with respect to $t$, which implies that $G_{12}(t, s) \leq G_{12}(e, s)$. Hence, inequality (ii) is proved.

(iii) Let $1 \leq t \leq s \leq e$ and $t \in I$. Then

$$
G_{11}(t, s)=\frac{1}{\Delta_{1}}\left[\eta_{1}(1-\ln s)^{-\gamma_{1}}+\frac{\xi_{1} \Gamma\left(\alpha_{1}-\gamma_{1}\right)}{\Gamma\left(\alpha_{1}\right)}\right](\ln t)^{\alpha_{1}-1}(1-\ln s)^{\alpha_{1}-1}
$$




$$
\begin{aligned}
& =\frac{(\ln t)^{\alpha_{1}-1}}{\Delta_{1}}\left[\eta_{1}(1-\ln s)^{-\gamma_{1}}+\frac{\xi_{1} \Gamma\left(\alpha_{1}-\gamma_{1}\right)}{\Gamma\left(\alpha_{1}\right)}\right](1-\ln s)^{\alpha_{1}-1} \\
& \geq\left(\frac{1}{4}\right)^{\alpha_{1}-1} G_{11}(e, s) .
\end{aligned}
$$

Let $1 \leq s \leq t \leq e$ and $t \in I$

$$
\begin{aligned}
G_{12}(t, s)= & \frac{1}{\Delta_{1}}\left[\eta_{1}(1-\ln s)^{-\gamma_{1}}+\frac{\xi_{1} \Gamma\left(\alpha_{1}-\gamma_{1}\right)}{\Gamma\left(\alpha_{1}\right)}\right](\ln t)^{\alpha_{1}-1}(1-\ln s)^{\alpha_{1}-1} \\
& -\frac{1}{\Gamma\left(\alpha_{1}\right)}\left(\ln \frac{t}{s}\right)^{\alpha_{1}-1} \\
= & \frac{1}{\Delta_{1}}\left[\eta(1-\ln s)^{-\gamma_{1}}+\frac{\xi_{1} \Gamma\left(\alpha_{1}-\gamma_{1}\right)}{\Gamma\left(\alpha_{1}\right)}\right](\ln t)^{\alpha_{1}-1}(1-\ln s)^{\alpha_{1}-1} \\
& -\frac{1}{\Gamma\left(\alpha_{1}\right)}\left(1-\frac{\ln s}{\ln t}\right)^{\alpha_{1}-1}(\ln t)^{\alpha_{1}-1} \\
\geq & (\ln t)^{\alpha_{1}-1}\left[\frac{1}{\Delta_{1}}\left(\eta_{1}(1-\ln s)^{-\gamma_{1}}+\frac{\xi_{1} \Gamma\left(\alpha_{1}-\gamma_{1}\right)}{\Gamma\left(\alpha_{1}\right)}\right)(1-\ln s)^{\alpha_{1}-1}\right. \\
& \left.-\frac{1}{\Gamma\left(\alpha_{1}\right)}(1-\ln s)^{\alpha_{1}-1}\right] \\
\geq & \left(\frac{1}{4}\right)^{\alpha_{1}-1} G_{12}(e, s) .
\end{aligned}
$$

Lemma 2.6 Assume that (A2) is satisfied. Then the Green's function $K_{1}(t, s)$ given by (6) satisfies the following inequalities:

(i) $0 \leq K_{1}(t, s) \leq l_{1}(s)$ for all $(t, s) \in[1, e] \times[1, e]$, where

$$
l_{1}(s)=K_{11}(s, s)+\frac{\vartheta_{1}}{1-\vartheta_{1}(\ln \delta)^{\beta_{1}-1}} K_{11}(\delta, s)
$$

(ii) $K_{1}(t, s) \geq \zeta_{1}(s) K_{11}(s, s)$ for all $(t, s) \in I \times(1, e)$, and $r \in I$,

$$
\zeta_{1}(s)= \begin{cases}\frac{\left(\frac{3}{4}\right)^{\beta_{1}-1}(1-\ln s)^{\beta_{1}-1}-\left(\frac{3}{4}-\ln s\right)^{\beta_{1}-1}}{(1-\ln s)^{\beta_{1}-1}}, & s \in(1, r], \\ \frac{1}{(4 \ln s)^{\beta_{1}-1}}, & s \in[r, e) .\end{cases}
$$

We can also formulate similar results as Lemmas 2.3-2.6 for the Hadamard fractional boundary value problems

$$
\left\{\begin{array}{l}
{ }^{H} D_{1^{+}}^{\beta_{2}}\left(\phi_{p_{2}}\left({ }^{H} D_{1^{+}}^{\alpha_{2}} y(t)\right)\right)=\mu g(t, x(t), y(t), z(t)), \quad t \in(1, e), \\
y(1)=y^{\prime}(1)=0 ; \quad \xi_{2} y(e)+\eta_{2}^{H} D_{1^{+}}^{\gamma_{2}} y(e)=0, \\
\phi_{p_{2}}\left({ }^{H} D_{1^{+}}^{\alpha_{2}} y(1)\right)=0, \quad \phi_{p_{2}}\left({ }^{H} D_{1^{+}}^{\alpha_{2}} y(e)\right)=\vartheta_{2} \phi_{p_{2}}\left({ }^{H} D_{1^{+}}^{\alpha_{2}} y(\delta)\right)
\end{array}\right.
$$

and

$$
\left\{\begin{array}{l}
{ }^{H} D_{1^{+}}^{\beta_{3}}\left(\phi_{p_{3}}\left({ }^{H} D_{1^{+}}^{\alpha_{3}} z(t)\right)\right)=v h(t, x(t), y(t), z(t)), \quad t \in(1, e), \\
z(1)=z^{\prime}(1)=0 ; \quad \xi_{3} z(e)+\eta_{3}^{H} D_{1^{+}}^{\gamma_{3}} z(e)=0, \\
\phi_{p_{3}}\left({ }^{H} D_{1^{+}}^{\alpha_{3}} z(1)\right)=0, \quad \phi_{p_{3}}\left({ }^{H} D_{1^{+}}^{\alpha_{3}} z(e)\right)=\vartheta_{3} \phi_{p_{3}}\left({ }^{H} D_{1^{+}}^{\alpha_{3}} z(\delta)\right) .
\end{array}\right.
$$


We also formulate the results of the Green's function $G_{i}(t, s)$ and $K_{i}(t, s), i=2,3$, for the homogeneous BVPs corresponding to the Hadamard fractional differential equations (7) and (8) and define it in a similar manner as $G_{1}(t, s)$ and $K_{1}(t, s)$.

Remark Consider the following conditions:

(i) $G_{i}(t, s) \geq m G_{i}(e, s)$ for all $(t, s) \in I \times(1, e), i=1,2,3$;

(ii) $K_{i}(t, s) \geq \zeta(s) K_{i 1}(e, s)$ for all $(t, s) \in I \times(1, e), i=1,2,3$, where $I=\left[e^{1 / 4}, e^{3 / 4}\right], m=\min \left\{\left(\frac{1}{4}\right)^{\alpha_{1}-1},\left(\frac{1}{4}\right)^{\alpha_{2}-1},\left(\frac{1}{4}\right)^{\alpha_{3}-1}\right\}, \zeta(s)=\min \left\{\zeta_{1}(s), \zeta_{2}(s), \zeta_{3}(s)\right\}$.

Our main results are based on the following Guo-Krasnosel'skii fixed-point theorem on cones.

Theorem 2.7 (Krasnosel'skii $[55,56])$ Let $X$ be a Banach space, $K \subseteq X$ be a cone, and suppose that $\Omega_{1}, \Omega_{2}$ are open subsets of $X$ with $0 \in \Omega_{1}$ and $\bar{\Omega}_{1} \subset \Omega_{2}$. Suppose further that $T: K \cap\left(\bar{\Omega}_{2} \backslash \Omega_{1}\right) \rightarrow K$ is a completely continuous operator such that either

(i) $\|T u\| \leq\|u\|, u \in K \cap \partial \Omega_{1}$ and $\|T u\| \geq\|u\|, u \in K \cap \partial \Omega_{2}$, or

(ii) $\|T u\| \geq\|u\|, u \in K \cap \partial \Omega_{1}$ and $\|T u\| \leq\|u\|, u \in K \cap \partial \Omega_{2}$

holds. Then $T$ has a fixed point in $K \cap\left(\bar{\Omega}_{2} \backslash \Omega_{1}\right)$.

\section{Multiplicity results}

In this section we investigate the existence of multiple positive solutions of problem (1)(2) under some assumptions on the functions $f, g$, and $h$ by establishing in the same time various intervals for the positive parameters $\lambda, \mu$, and $v$.

Let $X=C[1, e]$, then $X$ is a Banach space with the norm $\|x\|=\max _{t \in[1, e]}|x(t)|$. Let $Y=$ $X \times X \times X$, then $Y$ is a Banach space with the norm $\|(x, y, z)\|_{Y}=\|x\|+\|y\|+\|z\|$.

Define a cone $\mathcal{P} \subset Y$ by

$$
\begin{aligned}
\mathcal{P}= & \{(x, y, z) \in Y: x(t) \geq 0, y(t) \geq 0, z(t) \geq 0, \forall t \in[1, e], \\
& \left.\min _{t \in I}\{x(t)+y(t)+z(t)\} \geq m\|(x, y, z)\|_{Y}\right\},
\end{aligned}
$$

where $I=\left[e^{1 / 4}, e^{3 / 4}\right]$. For $\lambda, \mu, v>0$, we define now the operator $Q: \mathcal{P} \rightarrow Y$ by $Q(x, y, z)=$ $\left(Q_{\lambda}(x, y, z), Q_{\mu}(x, y, z), Q_{v}(x, y, z)\right)$ with

$$
\begin{aligned}
& Q_{\lambda}(x, y, z)(t)=\lambda^{q_{1}-1} \int_{1}^{e} G_{1}(t, s) \phi_{q_{1}}\left(\int_{1}^{e} K_{1}(s, \tau) f(\tau, x(\tau), y(\tau), z(\tau)) \frac{d \tau}{\tau}\right) \frac{d s}{s}, \\
& t \in[1, e], \\
& Q_{\mu}(u, v, w)(t)=\mu^{q_{2}-1} \int_{1}^{e} G_{2}(t, s) \phi_{q_{2}}\left(\int_{1}^{e} K_{2}(s, \tau) g(\tau, u(\tau), v(\tau), w(\tau)) \frac{d \tau}{\tau}\right) \frac{d s}{s}, \\
& \quad t \in[1, e], \\
& Q_{\nu}(u, v, w)(t)=v^{q_{3}-1} \int_{1}^{e} G_{3}(t, s) \phi_{q_{3}}\left(\int_{1}^{e} K_{3}(s, \tau) h(\tau, u(\tau), v(\tau), w(\tau)) \frac{d \tau}{\tau}\right) \frac{d s}{s}, \\
& t \in[1, e] .
\end{aligned}
$$

Lemma 3.1 $Q: \mathcal{P} \rightarrow \mathcal{P}$ is a completely continuous operator. 
Proof The continuity of functions $G_{i}(t, s), K_{i}(t, s), i=1,2,3$, and $f, g, h$ implies that $Q: \mathcal{P} \rightarrow$ $\mathcal{P}$ is continuous. For all $(t, s) \in I \times[1, e]$, where $I=\left[e^{1 / 4}, e^{3 / 4}\right]$, we have

$$
\begin{aligned}
\min _{t \in I}\left\{Q_{\lambda}(x, y, z)(t)+Q_{\mu}(x, y, z)(t)+Q_{v}(x, y, z)(t)\right\} \\
=\min _{t \in I}\left\{\lambda^{q_{1}-1} \int_{1}^{e} G_{1}(t, s) \phi_{q_{1}}\left(\int_{1}^{e} K_{1}(s, \tau) f(\tau, x(\tau), y(\tau), z(\tau)) \frac{d \tau}{\tau}\right) \frac{d s}{s}\right. \\
+\mu^{q_{2}-1} \int_{1}^{e} G_{2}(t, s) \phi_{q_{2}}\left(\int_{1}^{e} K_{2}(s, \tau) g(\tau, x(\tau), y(\tau), z(\tau)) \frac{d \tau}{\tau}\right) \frac{d s}{s} \\
\left.+v^{q_{3}-1} \int_{1}^{e} G_{3}(t, s) \phi_{q_{3}}\left(\int_{1}^{e} K_{3}(s, \tau) h(\tau, x(\tau), y(\tau), z(\tau)) \frac{d \tau}{\tau}\right) \frac{d s}{s}\right\} \\
\geq m\left\{\lambda^{q_{1}-1} \int_{1}^{e} G_{1}(e, s) \phi_{q_{1}}\left(\int_{1}^{e} K_{1}(s, \tau) f(\tau, x(\tau), y(\tau), z(\tau)) \frac{d \tau}{\tau}\right) \frac{d s}{s}\right. \\
+\mu^{q_{2}-1} \int_{1}^{e} G_{2}(e, s) \phi_{q_{2}}\left(\int_{1}^{e} K_{2}(s, \tau) g(\tau, x(\tau), y(\tau), z(\tau)) \frac{d \tau}{\tau}\right) \frac{d s}{s} \\
\left.+v^{q_{3}-1} \int_{1}^{e} G_{3}(e, s) \phi_{q_{3}}\left(\int_{1}^{e} K_{3}(s, \tau) h(\tau, x(\tau), y(\tau), z(\tau)) \frac{d \tau}{\tau}\right) \frac{d s}{s}\right\} \\
\geq m\left(\left\|Q_{\lambda}(x, y, z)\right\|+\left\|Q_{\mu}(x, y, z)\right\|+\left\|Q_{v}(x, y, z)\right\|\right) \\
=m\left\|\left(Q_{\lambda}(x, y, z), Q_{\mu}(x, y, z), Q_{v}(x, y, z)\right)\right\| \\
=m \| Q_{(x, y, z) \| .}
\end{aligned}
$$

Thus $Q(\mathcal{P}) \subset \mathcal{P}$. So, we can easily show that $Q: \mathcal{P} \rightarrow \mathcal{P}$ is a completely continuous operator by the Arzela-Ascoli theorem.

If $(x, y, z) \in \mathcal{P}$ is a fixed point of operator $Q$, then $(x, y, z)$ is a solution of problem (1)-(2). So, we will investigate the existence of fixed points of operator $Q$.

Theorem 3.2 Assume that conditions (A1)-(A4) are satisfied. In addition, assume that there exist constants $r_{1}, M, K, \zeta_{1}, \zeta_{2}, \zeta_{3}$, where $K$ is sufficiently small, $\zeta_{1}+\zeta_{2}+\zeta_{3}=1$, with $\left(\zeta_{1} m \rho_{1}\right)^{p_{1}-1} M>\left(\sigma_{1}\right)^{p_{1}-1} K,\left(\zeta_{2} m \rho_{2}\right)^{p_{2}-1} M>\left(\sigma_{2}\right)^{p_{2}-1} K,\left(\zeta_{3} m \rho_{3}\right)^{p_{3}-1} M>\left(\sigma_{3}\right)^{p_{3}-1} K$ such that:

(1) $u_{10}=u_{1 \infty}=0, u_{20}=u_{2 \infty}=0, u_{30}=u_{3 \infty}=0$;

(2) $f(t, x, y, z) \geq M\left(r_{1}\right)^{p_{1}-1}$, or $g(t, x, y, z) \geq M\left(r_{1}\right)^{p_{2}-1}$, or $h(t, x, y, z) \geq M\left(r_{1}\right)^{p_{3}-1}$ for $m r_{1} \leq\|(x, y, z)\|_{Y}<r_{1}$.

Then, for any

$$
\begin{aligned}
& \lambda \in\left[\frac{1}{M}\left(\frac{1}{m \rho_{1}}\right)^{p_{1}-1}, \frac{1}{K}\left(\frac{\zeta_{1}}{\sigma_{1}}\right)^{p_{1}-1}\right], \quad \mu \in\left(0, \frac{1}{K}\left(\frac{\zeta_{2}}{\sigma_{2}}\right)^{p_{2}-1}\right], \\
& \nu \in\left(0, \frac{1}{K}\left(\frac{\zeta_{3}}{\sigma_{3}}\right)^{p_{3}-1}\right], \quad \text { or } \\
& \lambda \in\left(0, \frac{1}{K}\left(\frac{\zeta_{1}}{\sigma_{1}}\right)^{p_{1}-1}\right], \quad \mu \in\left[\frac{1}{M}\left(\frac{1}{m \rho_{2}}\right)^{p_{2}-1}, \frac{1}{K}\left(\frac{\zeta_{2}}{\sigma_{2}}\right)^{p_{2}-1}\right], \\
& \nu \in\left(0, \frac{1}{K}\left(\frac{\zeta_{3}}{\sigma_{3}}\right)^{p_{3}-1}\right], \quad \text { or }
\end{aligned}
$$




$$
\begin{aligned}
& \lambda \in\left(0, \frac{1}{K}\left(\frac{\zeta_{1}}{\sigma_{1}}\right)^{p_{1}-1}\right], \quad \mu \in\left(0, \frac{1}{K}\left(\frac{\zeta_{2}}{\sigma_{2}}\right)^{p_{2}-1}\right], \\
& \nu \in\left[\frac{1}{M}\left(\frac{1}{m \rho_{3}}\right)^{p_{3}-1}, \frac{1}{K}\left(\frac{\zeta_{3}}{\sigma_{3}}\right)^{p_{3}-1}\right],
\end{aligned}
$$

system (1)-(2) has at least two positive solutions.

Proof We only prove the case of $\lambda \in\left[\frac{1}{M}\left(\frac{1}{m \rho_{1}}\right)^{p_{1}-1}, \frac{1}{K}\left(\frac{\zeta_{1}}{\sigma_{1}}\right)^{p_{1}-1}\right], \mu \in\left(0, \frac{1}{K}\left(\frac{\zeta_{2}}{\sigma_{2}}\right)^{p_{2}-1}\right], v \in$ $\left(0, \frac{1}{K}\left(\frac{\zeta_{3}}{\sigma_{3}}\right)^{p_{3}-1}\right]$. The other cases are similar.

Step 1 . By the definition of $u_{10}=u_{20}=u_{30}=0$, there exists $H_{1} \in\left(0, r_{1}\right)$ such that

$$
\begin{array}{ll}
u_{1}(t, x, y, z) \leq K(x+y+z)^{p_{1}-1} & \text { for } x+y+z \in\left(0, H_{1}\right), \\
u_{2}(t, x, y, z) \leq K(x+y+z)^{p_{2}-1} & \text { for } x+y+z \in\left(0, H_{1}\right), \\
u_{3}(t, x, y, z) \leq K(x+y+z)^{p_{3}-1} & \text { for } x+y+z \in\left(0, H_{1}\right) .
\end{array}
$$

Then we have

$$
\begin{aligned}
Q_{\lambda} & (x, y, z)(t) \\
& =\lambda^{q_{1}-1} \int_{1}^{e} G_{1}(t, s) \phi_{q_{1}}\left(\int_{1}^{e} K_{1}(s, \tau) f(\tau, u(\tau), v(\tau), w(\tau)) \frac{d \tau}{\tau}\right) \frac{d s}{s} \\
& \leq \lambda^{q_{1}-1} \int_{1}^{e} G_{1}(e, s) \phi_{q_{1}}\left(\int_{1}^{e} K_{1}(s, \tau) \kappa_{1}(\tau) u_{1}(\tau, x(\tau), y(\tau), z(\tau)) \frac{d \tau}{\tau}\right) \frac{d s}{s} \\
& \leq \lambda^{q_{1}-1} \int_{1}^{e} G_{1}(e, s) \phi_{q_{1}}\left(\int_{1}^{e} l_{1}(\tau) \kappa_{1}(\tau) K(x(\tau)+y(\tau)+z(\tau))^{p_{1}-1} \frac{d \tau}{\tau}\right) \frac{d s}{s} \\
& \leq \lambda^{q_{1}-1} K^{q_{1}-1}\|(x, y, z)\|_{Y} \int_{1}^{e} G_{1}(e, s) \phi_{q_{1}}\left(\int_{1}^{e} l_{1}(\tau) \kappa_{1}(\tau) \frac{d \tau}{\tau}\right) \frac{d s}{s} \\
& \leq(\lambda K)^{q_{1}-1}\|(x, y, z)\|_{Y} \sigma_{1} \leq \zeta_{1}\|(x, y, z)\|_{Y}, \\
Q_{\mu}(x, y, z)(t) & \\
& =\mu^{q_{2}-1} \int_{1}^{e} G_{2}(t, s) \phi_{q_{2}}\left(\int_{1}^{e} K_{2}(s, \tau) g(\tau, u(\tau), v(\tau), w(\tau)) \frac{d \tau}{\tau}\right) \frac{d s}{s} \\
& \leq \mu^{q_{2}-1} \int_{1}^{e} G_{2}(e, s) \phi_{q_{2}}\left(\int_{1}^{e} K_{2}(s, \tau) \kappa_{2}(\tau) u_{2}(\tau, x(\tau), y(\tau), z(\tau)) \frac{d \tau}{\tau}\right) \frac{d s}{s} \\
& \leq \mu^{q_{2}-1} \int_{1}^{e} G_{2}(e, s) \phi_{q_{2}}\left(\int_{1}^{e} l_{2}(\tau) \kappa_{2}(\tau) K(x(\tau)+y(\tau)+z(\tau))^{p_{2}-1} \frac{d \tau}{\tau}\right) \frac{d s}{s} \\
& \leq \mu^{q_{2}-1} K^{q_{2}-1}\|(x, y, z)\|_{Y} \int_{1}^{e} G_{2}(e, s) \phi_{q_{2}}\left(\int_{1}^{e} l_{2}(\tau) \kappa_{2}(\tau) \frac{d \tau}{\tau}\right) \frac{d s}{s} \\
& \leq(\mu K)^{q_{2}-1}\|(x, y, z)\|_{Y} \sigma_{2} \leq \zeta_{2}\|(x, y, z)\|_{Y}, \\
Q_{\nu}(x, y, z)(t) & v^{q_{3}-1} \int_{1}^{e} G_{3}(t, s) \phi_{q_{3}}\left(\int_{1}^{e} K_{3}(s, \tau) h(\tau, u(\tau), v(\tau), w(\tau)) \frac{d \tau}{\tau}\right) \frac{d s}{s} \\
&
\end{aligned}
$$




$$
\begin{aligned}
& \leq v^{q_{3}-1} \int_{1}^{e} G_{3}(e, s) \phi_{q_{3}}\left(\int_{1}^{e} l_{3}(\tau) \kappa_{3}(\tau) K(x(\tau)+y(\tau)+z(\tau))^{p_{3}-1} \frac{d \tau}{\tau}\right) \frac{d s}{s} \\
& \leq v^{q_{3}-1} K^{q_{3}-1}\|(x, y, z)\|_{Y} \int_{1}^{e} G_{3}(e, s) \phi_{q_{3}}\left(\int_{1}^{e} l_{3}(\tau) \kappa_{3}(\tau) \frac{d \tau}{\tau}\right) \frac{d s}{s} \\
& \leq(\nu K)^{q_{3}-1}\|(x, y, z)\|_{Y} \sigma_{3} \leq \zeta_{3}\|(x, y, z)\|_{Y} .
\end{aligned}
$$

Hence,

$$
\begin{aligned}
\|Q(x, y, z)\| & =\left\|\left(Q_{\lambda}(x, y, z), Q_{\mu}(x, y, z), Q_{v}(x, y, z)\right)\right\| \\
& =\left\|Q_{\lambda}(x, y, z)\right\|+\left\|Q_{\mu}(x, y, z)\right\|+\left\|Q_{v}(x, y, z)\right\| \\
& \leq \zeta_{1}\|(x, y, z)\|_{Y}+\zeta_{2}\|(x, y, z)\|_{Y}+\zeta_{3}\|(x, y, z)\|_{Y} \\
& =\left(\zeta_{1}+\zeta_{2}+\zeta_{3}\right)\|(x, y, z)\|_{Y}=\|(x, y, z)\|_{Y} .
\end{aligned}
$$

Consequently, if we set $\Omega_{1}=\left\{(x, y, z) \in \mathcal{P}:\|(x, y, z)\|_{Y}<H_{1}\right\}$, then

$$
\|Q(x, y, z)\| \leq\|(x, y, z)\|_{Y} \quad \text { for all }(x, y, z) \in \mathcal{P} \cap \partial \Omega_{1} .
$$

Step 2. By the definition of $u_{1 \infty}=u_{2 \infty}=u_{3 \infty}=0$, there exists $H_{2}>r_{1}$ such that

$$
\begin{array}{ll}
u_{1}(t, x, y, z) \leq K(x+y+z)^{p_{1}-1} & \text { for } x+y+z \in\left[H_{2}, \infty\right), \\
u_{2}(t, x, y, z) \leq K(x+y+z)^{p_{2}-1} & \text { for } x+y+z \in\left[H_{2}, \infty\right), \\
u_{3}(t, x, y, z) \leq K(x+y+z)^{p_{3}-1} & \text { for } x+y+z \in\left[H_{2}, \infty\right) .
\end{array}
$$

Similarly, set $\Omega_{2}=\left\{(x, y, z) \in \mathcal{P}:\|(x, y, z)\|_{Y}<H_{2}\right\}$, then

$$
\|Q(x, y, z)\| \leq\|(x, y, z)\|_{Y} \quad \text { for all }(x, y, z) \in \mathcal{P} \cap \partial \Omega_{2}
$$

Step 3. Set $\Omega_{3}=\left\{(x, y, z) \in \mathcal{P}:\|(x, y, z)\|_{Y}<r_{1}\right\}$, then for all $(x, y, z) \in \mathcal{P}$ with $\|(x, y, z)\|_{Y}=r_{1}$, we have

$$
\begin{aligned}
Q_{\lambda}(x, y, z)(t) & =\lambda^{q_{1}-1} \int_{1}^{e} G_{1}(t, s) \phi_{q_{1}}\left(\int_{1}^{e} K_{1}(s, \tau) f(\tau, u(\tau), v(\tau), w(\tau)) \frac{d \tau}{\tau}\right) \frac{d s}{s} \\
& \geq \lambda^{q_{1}-1} m \int_{s \in I} G_{1}(e, s) \phi_{q_{1}}\left(\int_{1}^{e} K_{1}(s, \tau) M\left(r_{1}\right)^{p_{1}-1} \frac{d \tau}{\tau}\right) \frac{d s}{s} \\
& \geq \lambda^{q_{1}-1} m M^{q_{1}-1} l_{1} \int_{s \in I} G_{1}(e, s)\left(\int_{s \in I} \varsigma(\tau) K_{11}(\tau, \tau)\right) \frac{d s}{s} \\
& =(\lambda M)^{q_{1}-1} m l_{1} \rho_{1} \geq r_{1} \quad \forall t \in I .
\end{aligned}
$$

Then

$$
\|Q(x, y, z)\| \geq\|(x, y, z)\|_{Y} \quad \text { for all }(x, y, z) \in \mathcal{P} \cap \partial \Omega_{3}
$$

Consequently, from (9)-(11) and Theorem 2.7, the system has at least two positive solutions $\left(x_{1}, y_{1}, z_{1}\right) \in \mathcal{P},\left(x_{2}, y_{2}, z_{2}\right) \in \mathcal{P}$ with $0<\left\|\left(x_{1}, y_{1}, z_{1}\right)\right\|_{Y}<r_{1}<\left\|\left(x_{2}, y_{2}, z_{2}\right)\right\|_{Y}$. 
The following result is an antithesis of Theorem 3.2.

Theorem 3.3 Assume that conditions (A1)-(A4) are satisfied. In addition, assume that there exist four constants $r_{1}, M, K, \varrho_{1}, \varrho_{2}, \varrho_{3}$, where $K$ is sufficiently large, $\varrho_{1}+\varrho_{2}+\varrho_{3}=$ 1 , with $\left(\varrho_{1} m \rho_{1}\right)^{p_{1}-1} K>\left(\sigma_{1}\right)^{p_{1}-1} M,\left(\varrho_{2} m \rho_{2}\right)^{p_{2}-1} K>\left(\sigma_{2}\right)^{p_{2}-1} M,\left(\varrho_{3} m \rho_{3}\right)^{p_{3}-1} K>\left(\sigma_{3}\right)^{p_{3}-1} M$ such that

(3) $u_{1}(t, x, y, z) \leq M\left(r_{1}\right)^{p_{1}-1}$, or $u_{2}(t, x, y, z) \leq M\left(r_{1}\right)^{p_{2}-1}$, or $u_{3}(t, x, y, z) \leq M\left(r_{1}\right)^{p_{3}-1}$ for $0 \leq\|(x, y, z)\|_{Y} \leq r_{1}$

(4) $f_{0}=f_{\infty}=\infty$ or $g_{0}=g_{\infty}=\infty$ or $h_{0}=h_{\infty}=\infty$.

Then, for any

$$
\begin{aligned}
& \lambda \in\left[\frac{1}{K}\left(\frac{1}{m \rho_{1}}\right)^{p_{1}-1}, \frac{1}{M}\left(\frac{\varrho_{1}}{\sigma_{1}}\right)^{p_{1}-1}\right], \quad \mu \in\left(0, \frac{1}{M}\left(\frac{\varrho_{2}}{\sigma_{2}}\right)^{p_{2}-1}\right], \\
& \nu \in\left(0, \frac{1}{M}\left(\frac{\varrho_{3}}{\sigma_{3}}\right)^{p_{3}-1}\right], \quad \text { or } \\
& \lambda \in\left(0, \frac{1}{M}\left(\frac{\varrho_{1}}{\sigma_{1}}\right)^{p_{1}-1}\right], \quad \mu \in\left[\frac{1}{K}\left(\frac{1}{m \rho_{2}}\right)^{p_{2}-1}, \frac{1}{M}\left(\frac{\varrho_{2}}{\sigma_{2}}\right)^{p_{2}-1}\right], \\
& \nu \in\left(0, \frac{1}{M}\left(\frac{\varrho_{3}}{\sigma_{3}}\right)^{p_{3}-1}\right], \quad \text { or } \\
& \lambda \in\left(0, \frac{1}{M}\left(\frac{\varrho_{1}}{\sigma_{1}}\right)^{p_{1}-1}\right], \quad \mu \in\left(0, \frac{1}{M}\left(\frac{\varrho_{2}}{\sigma_{2}}\right)^{p_{2}-1}\right], \\
& \nu \in\left[\frac{1}{K}\left(\frac{1}{m \rho_{3}}\right)^{p_{3}-1}, \frac{1}{M}\left(\frac{\varrho_{3}}{\sigma_{3}}\right)^{p_{3}-1}\right],
\end{aligned}
$$

system (1)-(2) has at least two positive solutions.

For the convenience of the discussion of more than two positive solutions for system (1)(2), we study the problem under a more general case than the assumption of Theorem 3.2 and Theorem 3.3.

$$
\begin{aligned}
& \varphi_{i}(r)=\sup \left\{q_{i}(t, x, y, z): t \in[1, e], m r \leq x+y+z \leq r\right\}, \quad i=1,2,3, \\
& \psi_{1}(r)=\inf \{f(t, x, y, z): t \in I, m r \leq x+y+z \leq r\}, \\
& \psi_{2}(r)=\inf \{g(t, x, y, z): t \in I, m r \leq x+y+z \leq r\}, \\
& \psi_{3}(r)=\inf \{h(t, x, y, z): t \in I, m r \leq x+y+z \leq r\}, \\
& \varphi(r)=\max \left\{\varphi_{1}(r), \varphi_{2}(r), \varphi_{3}(r)\right\}, \quad \psi(r)=\min \left\{\psi_{1}(r), \psi_{2}(r), \psi_{3}(r)\right\} .
\end{aligned}
$$

Then, we can obtain the following result.

Theorem 3.4 Assume that conditions (A1)-(A4) are satisfied. In addition, assume that there exist three constants $M, K, \varsigma_{1}, \varsigma_{2}, \varsigma_{3}$ and $\varsigma_{1}+\varsigma_{2}+\varsigma_{3}=1$ with $\left(\varsigma_{1} m \rho_{1}\right)^{p_{1}-1} M>$ $\left(\sigma_{1}\right)^{p_{1}-1} K,\left(\varsigma_{2} m \rho_{2}\right)^{p_{2}-1} M>\left(\sigma_{2}\right)^{p_{2}-1} K,\left(\varsigma_{3} m \rho_{3}\right)^{p_{3}-1} M>\left(\sigma_{3}\right)^{p_{3}-1} K$ and three constants $d_{1}, d_{2}$, $d_{3}$ with $0<d_{1}<d_{2}<d_{3}$, such that one of the following two conditions is satisfied:

(I) $\varphi\left(d_{1}\right) \leq K\left(d_{1}\right)^{p_{i}-1}, \psi\left(d_{2}\right)>M\left(d_{2}\right)^{p_{i}-1}$, and $\varphi\left(d_{3}\right) \leq K\left(d_{3}\right)^{p_{i}-1}, i=1,2,3$;

(II) $\psi\left(d_{1}\right) \geq M\left(d_{1}\right)^{p_{i}-1}, \varphi\left(d_{2}\right)<K\left(d_{2}\right)^{p_{i}-1}$, and $\psi\left(d_{3}\right) \geq M\left(d_{3}\right)^{p_{i}-1}, i=1,2,3$. 
Then, for any

$$
\begin{aligned}
& \lambda \in\left[\frac{1}{M}\left(\frac{1}{m \rho_{1}}\right)^{p_{1}-1}, \frac{1}{K}\left(\frac{\varsigma_{1}}{\sigma_{1}}\right)^{p_{1}-1}\right], \quad \mu \in\left(0, \frac{1}{K}\left(\frac{\varsigma_{2}}{\sigma_{2}}\right)^{p_{2}-1}\right], \\
& \nu \in\left(0, \frac{1}{K}\left(\frac{\varsigma_{3}}{\sigma_{3}}\right)^{p_{3}-1}\right], \quad \text { or } \\
& \lambda \in\left(0, \frac{1}{K}\left(\frac{\varsigma_{1}}{\sigma_{1}}\right)^{p_{1}-1}\right], \quad \mu \in\left[\frac{1}{M}\left(\frac{1}{m \rho_{2}}\right)^{p_{2}-1}, \frac{1}{K}\left(\frac{\varsigma_{2}}{\sigma_{2}}\right)\right], \\
& \nu \in\left(0, \frac{1}{K}\left(\frac{\varsigma_{3}}{\sigma_{3}}\right)^{p_{2}-1}\right], \quad \text { or } \\
& \lambda \in\left(0, \frac{1}{K}\left(\frac{\varsigma_{1}}{\sigma_{1}}\right)^{p_{1}-1}\right], \quad \mu \in\left(0, \frac{1}{K}\left(\frac{\varsigma_{2}}{\sigma_{2}}\right)^{p_{2}-1}\right], \\
& \mu \in\left[\frac{1}{M}\left(\frac{1}{m \rho_{3}}\right)^{p_{3}-1}, \frac{1}{K}\left(\frac{\varsigma_{3}}{\sigma_{3}}\right)^{p_{3}-1}\right],
\end{aligned}
$$

system (1)-(2) has at least two positive solutions $\left(x_{1}^{\star}, y_{1}^{\star}, z_{1}^{\star}\right),\left(x_{2}^{\star}, y_{2}^{\star}, z_{2}^{\star}\right)$ and $d_{1} \leq \|\left(x_{1}^{\star}, y_{1}^{\star}\right.$, $\left.z_{1}^{\star}\right)\left\|_{Y}<d_{2}<\right\|\left(x_{2}^{\star}, y_{2}^{\star}, z_{2}^{\star}\right) \|_{Y} \leq d_{3}$.

Proof We only prove the case of $(I)$ and $\lambda \in\left[\frac{1}{M}\left(\frac{1}{m \rho_{1}}\right)^{p_{1}-1}, \frac{1}{K}\left(\frac{\varsigma 1}{\sigma_{1}}\right)^{p_{1}-1}\right], \mu \in\left(0, \frac{1}{K}\left(\frac{\varsigma 2}{\sigma_{2}}\right)^{p_{2}-1}\right], v \in$ $\left(0, \frac{1}{K}\left(\frac{\varsigma 3}{\sigma_{3}}\right)^{p_{3}-1}\right]$. The other cases are similar. Let $\Omega_{d_{1}}=\left\{(x, y, z) \in \mathcal{P}:\|(x, y, z)\|_{Y}<d_{1}\right\}$. If $(x, y, z) \in \partial \Omega_{d_{1}}$, then $\|(x, y, z)\|_{Y}=d_{1}$. Since $m d_{1} \leq x+y+z \leq d_{1}, 1 \leq t \leq e$, then we have

$$
\begin{aligned}
Q_{\lambda} & (x, y, z)(t) \\
& =\lambda^{q_{1}-1} \int_{1}^{e} G_{1}(t, s) \phi_{q_{1}}\left(\int_{1}^{e} K_{1}(s, \tau) f(\tau, u(\tau), v(\tau), w(\tau)) \frac{d \tau}{\tau}\right) \frac{d s}{s} \\
& \leq \lambda^{q_{1}-1} \int_{1}^{e} G_{1}(e, s) \phi_{q_{1}}\left(\int_{1}^{e} K_{1}(s, \tau) \kappa_{1}(\tau) u_{1}(\tau, x(\tau), y(\tau), z(\tau)) \frac{d \tau}{\tau}\right) \frac{d s}{s} \\
& \leq \lambda^{q_{1}-1} \int_{1}^{e} G_{1}(e, s) \phi_{q_{1}}\left(\int_{1}^{e} l_{1}(\tau) \kappa_{1}(\tau) \varphi\left(d_{1}\right) \frac{d \tau}{\tau}\right) \frac{d s}{s} \\
& \leq \lambda^{q_{1}-1} K^{q_{1}-1} d_{1} \int_{1}^{e} G_{1}(e, s) \phi_{q_{1}}\left(\int_{1}^{e} l_{1}(\tau) \kappa_{1}(\tau) \frac{d \tau}{\tau}\right) \frac{d s}{s} \\
& =(\lambda K)^{q_{1}-1} d_{1} \sigma_{1} \leq d_{1} \varsigma_{1}=\varsigma_{1}\|(x, y, z)\|_{Y}, \\
Q_{\mu} & (x, y, z)(t) \\
& =\mu^{q_{2}-1} \int_{1}^{e} G_{2}(t, s) \phi_{q_{2}}\left(\int_{1}^{e} K_{2}(s, \tau) g(\tau, u(\tau), v(\tau), w(\tau)) \frac{d \tau}{\tau}\right) \frac{d s}{s} \\
& \leq(\mu K)^{q_{2}-1} d_{1} \sigma_{2} \leq d_{1} \varsigma_{2}=\varsigma_{2}\|(x, y, z)\|_{Y}, \\
& \leq \mu^{q_{2}-1} \int_{1}^{e} G_{2}(e, s) \phi_{q_{2}}\left(\int_{1}^{e} K_{2}(s, \tau) \kappa_{2}(\tau) u_{2}(\tau, x(\tau), y(\tau), z(\tau)) \frac{d \tau}{\tau}\right) \frac{d s}{s} \\
& \leq \mu^{q_{2}-1} \int_{1}^{e} G_{2}(e, s) \phi_{q_{2}}\left(\int_{1}^{e} l_{2}(\tau) \kappa_{2}(\tau) \varphi\left(d_{1}\right) \frac{d \tau}{\tau}\right) \frac{d s}{s} \\
& \leq \mu^{q_{2}-1} K^{q_{2}-1} d_{1} \int_{1}^{e} G_{2}(e, s) \phi_{q_{2}}\left(\int_{1}^{e} l_{2}(\tau) \kappa_{2}(\tau) \frac{d \tau}{\tau}\right) \frac{d s}{s} \\
&
\end{aligned}
$$




$$
\begin{aligned}
Q_{v} & (x, y, z)(t) \\
& =v^{q_{3}-1} \int_{1}^{e} G_{3}(t, s) \phi_{q_{3}}\left(\int_{1}^{e} K_{3}(s, \tau) h(\tau, u(\tau), v(\tau), w(\tau)) \frac{d \tau}{\tau}\right) \frac{d s}{s} \\
& \leq v^{q_{3}-1} \int_{1}^{e} G_{3}(e, s) \phi_{q_{3}}\left(\int_{1}^{e} K_{3}(s, \tau) \kappa_{3}(\tau) u_{3}(\tau, x(\tau), y(\tau), z(\tau)) \frac{d \tau}{\tau}\right) \frac{d s}{s} \\
& \leq v^{q_{3}-1} \int_{1}^{e} G_{3}(e, s) \phi_{q_{3}}\left(\int_{1}^{e} l_{3}(\tau) \kappa_{3}(\tau) \varphi\left(d_{1}\right) \frac{d \tau}{\tau}\right) \frac{d s}{s} \\
& \leq v^{q_{1}-1} K^{q_{1}-1} d_{1} \int_{1}^{e} G_{3}(e, s) \phi_{q_{3}}\left(\int_{1}^{e} l_{3}(\tau) \kappa_{3}(\tau) \frac{d \tau}{\tau}\right) \frac{d s}{s} \\
& =(\nu K)^{q_{3}-1} d_{1} \sigma_{3} \leq d_{1} \varsigma_{3}=\varsigma_{3}\|(x, y, z)\|_{Y^{\prime}} .
\end{aligned}
$$

Hence,

$$
\begin{aligned}
\|Q(x, y, z)\| & =\left\|\left(Q_{\lambda}(x, y, z), Q_{\mu}(x, y, z), Q_{\nu}(x, y, z)\right)\right\| \\
& =\left\|Q_{\lambda}(x, y, z)\right\|+\left\|Q_{\mu}(x, y, z)\right\|+\left\|Q_{\nu}(x, y, z)\right\| \\
& \leq \varsigma_{1}\|(x, y, z)\|_{Y}+\varsigma_{2}\|(x, y, z)\|_{Y}+\varsigma_{3}\|(x, y, z)\|_{Y} \\
& =\left(\varsigma_{1}+\varsigma_{2}+\varsigma_{3}\right)\|(x, y, z)\|_{Y}=\|(x, y, z)\|_{Y} .
\end{aligned}
$$

Then

$$
\|Q(x, y, z)\| \leq\|(x, y, z)\|_{Y}, \quad \text { for all }(x, y, z) \in \mathcal{P} \cap \partial \Omega_{d_{1}}
$$

Let $\Omega_{d_{2}}=\left\{(x, y, z) \in \mathcal{P}:\|(x, y, z)\|_{Y}<d_{2}\right\}$. If $(x, y, z) \in \partial \Omega_{d_{2}}$, then $\|(x, y, z)\|_{Y}=d_{2}$. Since $m d_{2} \leq x+y+z \leq d_{2}, t \in I$, then we have

$$
\begin{aligned}
Q_{\lambda}(x, y, z)(t) & =\lambda^{q_{1}-1} \int_{1}^{e} G_{1}(t, s) \phi_{q_{1}}\left(\int_{1}^{e} K_{1}(s, \tau) f(\tau, u(\tau), v(\tau), w(\tau)) \frac{d \tau}{\tau}\right) \frac{d s}{s} \\
& \geq \lambda^{q_{1}-1} m \int_{s \in I} G_{1}(e, s) \phi_{q_{1}}\left(\int_{1}^{e} K_{1}(s, \tau) \psi\left(d_{2}\right) \frac{d \tau}{\tau}\right) \frac{d s}{s} \\
& >\lambda^{q_{1}-1} m M^{q_{1}-1} d_{2} \int_{s \in I} G_{1}(e, s) \phi_{q_{1}}\left(\int_{s \in I} \varsigma(\tau) K_{11}(\tau, \tau) \frac{d \tau}{\tau}\right) \frac{d s}{s} \\
& =(\lambda M)^{q_{1}-1} m d_{2} \rho_{1} \geq d_{2}=\|(x, y, z)\|_{Y}, \quad \forall t \in I .
\end{aligned}
$$

Then

$$
\|Q(x, y, z)\| \geq\|(x, y, z)\|_{Y} \quad \text { for all }(x, y, z) \in \mathcal{P} \cap \partial \Omega_{d_{2}}
$$

Let $\Omega_{d_{3}}=\left\{(x, y, z) \in \mathcal{P}:\|(x, y, z)\|_{Y}<d_{3}\right\}$. If $(x, y, z) \in \partial \Omega_{d_{3}}$, then $\|(x, y, z)\|_{Y}=d_{3}$. Since $m d_{3} \leq x+y+z \leq d_{3}, 1 \leq t \leq e$, then we have

$$
\begin{aligned}
& Q_{\lambda}(x, y, z)(t) \\
& \quad=\lambda^{q_{1}-1} \int_{1}^{e} G_{1}(t, s) \phi_{q_{1}}\left(\int_{1}^{e} K_{1}(s, \tau) f(\tau, u(\tau), v(\tau), w(\tau)) \frac{d \tau}{\tau}\right) \frac{d s}{s}
\end{aligned}
$$




$$
\begin{aligned}
& \leq \lambda^{q_{1}-1} \int_{1}^{e} G_{1}(e, s) \phi_{q_{1}}\left(\int_{1}^{e} K_{1}(s, \tau) \kappa_{1}(\tau) u_{1}(\tau, x(\tau), y(\tau), z(\tau)) \frac{d \tau}{\tau}\right) \frac{d s}{s} \\
& \leq \lambda^{q_{1}-1} \int_{1}^{e} G_{1}(e, s) \phi_{q_{1}}\left(\int_{1}^{e} l_{1}(\tau) \kappa_{1}(\tau) \varphi\left(d_{3}\right) \frac{d \tau}{\tau}\right) \frac{d s}{s} \\
& \leq \lambda^{q_{1}-1} K^{q_{1}-1} d_{3} \int_{1}^{e} G_{1}(e, s) \phi_{q_{1}}\left(\int_{1}^{e} l_{1}(\tau) \kappa_{1}(\tau) \frac{d \tau}{\tau}\right) \frac{d s}{s} \\
& =(\lambda K)^{q_{1}-1} d_{3} \sigma_{1} \leq d_{3} S_{1}=s_{1}\|(x, y, z)\|_{Y}, \\
Q_{\mu} & (x, y, z)(t) \\
& =\mu^{q_{2}-1} \int_{1}^{e} G_{2}(t, s) \phi_{q_{2}}\left(\int_{1}^{e} K_{2}(s, \tau) g(\tau, u(\tau), v(\tau), w(\tau)) \frac{d \tau}{\tau}\right) \frac{d s}{s} \\
& \leq \mu^{q_{2}-1} \int_{1}^{e} G_{2}(e, s) \phi_{q_{2}}\left(\int_{1}^{e} K_{2}(s, \tau) \kappa_{2}(\tau) u_{2}(\tau, x(\tau), y(\tau), z(\tau)) \frac{d \tau}{\tau}\right) \frac{d s}{s} \\
& \leq \mu^{q_{2}-1} \int_{1}^{e} G_{2}(e, s) \phi_{q_{2}}\left(\int_{1}^{e} l_{2}(\tau) \kappa_{2}(\tau) \varphi\left(d_{3}\right) \frac{d \tau}{\tau}\right) \frac{d s}{s} \\
& \leq \mu^{q_{2}-1} K^{q_{2}-1} d_{1} \int_{1}^{e} G_{2}(e, s) \phi_{q_{2}}\left(\int_{1}^{e} l_{2}(\tau) \kappa_{2}(\tau) \frac{d \tau}{\tau}\right) \frac{d s}{s} \\
& =(\mu K)^{q_{2}-1} d_{3} \sigma_{2} \leq d_{3} \varsigma_{2}=\varsigma_{2}\|(x, y, z)\|_{Y}, \\
& \leq v^{q_{1}-1} K^{q_{1}-1} d_{3} \int_{1}^{e} G_{3}(e, s) \phi_{q_{3}}\left(\int_{1}^{e} l_{3}(\tau) \kappa_{3}(\tau) \frac{d \tau}{\tau}\right) \frac{d s}{s} \\
& =(v K)^{q_{3}-1} d_{3} \sigma_{3} \leq d_{3} \varsigma_{3}=s_{3}\|(x, y, z)\|_{Y} . \\
& =v^{q_{3}-1} \int_{1}^{q_{3}-1} \int_{1}^{e} G_{3}^{e} G_{3}(t, s) \phi_{q_{3}}\left(\int_{1}^{e} K_{3}(s, \tau) h(\tau, u(\tau), v(\tau), w(\tau)) \frac{d \tau}{\tau}\right) \frac{d s}{s} \\
&
\end{aligned}
$$

Then

$$
\begin{aligned}
\|Q(x, y, z)\| & =\left\|\left(Q_{\lambda}(x, y, z), Q_{\mu}(x, y, z), Q_{\nu}(x, y, z)\right)\right\| \\
& =\left\|Q_{\lambda}(x, y, z)\right\|+\left\|Q_{\mu}(x, y, z)\right\|+\left\|Q_{v}(x, y, z)\right\| \\
& \leq \varsigma_{1}\|(x, y, z)\|_{Y}+\varsigma_{2}\|(x, y, z)\|_{Y}+\varsigma_{3}\|(x, y, z)\|_{Y} \\
& =\left(\varsigma_{1}+\varsigma_{2}+\varsigma_{3}\right)\|(x, y, z)\|_{Y}=\|(x, y, z)\|_{Y} . \\
\|Q(x, y, z)\| & \leq\|(x, y, z)\|_{Y} \quad \text { for all }(x, y, z) \in \mathcal{P} \cap \partial \Omega_{d_{3}} .
\end{aligned}
$$

From (12)-(14) and Theorem 2.7, the system has at least two positive solutions $\left(x_{1}^{\star}, y_{1}^{\star}, z_{1}^{\star}\right) \in$ $\mathcal{P},\left(x_{2}^{\star}, y_{2}^{\star}, z_{2}^{\star}\right) \in \mathcal{P}$, and $d_{1} \leq\left\|\left(x_{1}^{\star}, y_{1}^{\star}, z_{1}^{\star}\right)\right\|_{Y}<d_{2}<\left\|\left(x_{2}^{\star}, y_{2}^{\star}, z_{2}^{\star}\right)\right\|_{Y} \leq d_{3}$.

Corollary 3.5 Assume that conditions (A1)-(A4) are satisfied, $\delta_{1}, \delta_{2}, \delta_{3}>0$ with $\delta_{1}+\delta_{2}+$ $\delta_{3}=1$, then we have the following results: 
(1) If $0<u_{10}, f_{\infty}, u_{20}, g_{\infty}, u_{30}, h_{\infty}<\infty,\left(\sigma_{1}\right)^{p_{1}-1} u_{10}<\left(\delta_{1} m^{2} \rho_{1}\right)^{p_{1}-1} f_{\infty}$, then for each $\lambda \in\left(\frac{1}{f_{\infty}}\left(\frac{1}{m^{2} \rho_{1}}\right)^{p_{1}-1}, \frac{1}{u_{10}}\left(\frac{\delta_{1}}{\sigma_{1}}\right)^{p_{1}-1}\right), \mu \in\left(0, \frac{1}{u_{20}}\left(\frac{\delta_{2}}{\sigma_{2}}\right)^{p_{2}-1}\right)$, and $v \in\left(0, \frac{1}{u_{30}}\left(\frac{\delta_{3}}{\sigma_{3}}\right)^{p_{3}-1}\right)$, system (1)-(2) has at least one positive solution.

(2) If $0<u_{10}, f_{\infty}, u_{20}, g_{\infty}, u_{30}, h_{\infty}<\infty,\left(\sigma_{2}\right)^{p_{2}-1} u_{20}<\left(\delta_{2} m^{2} \rho_{2}\right)^{p_{2}-1} g_{\infty}$, then for each $\lambda \in\left(0, \frac{1}{u_{10}}\left(\frac{\delta_{1}}{\sigma_{1}}\right)^{p_{1}-1}\right), \mu \in\left(\frac{1}{g_{\infty}}\left(\frac{1}{m^{2} \rho_{2}}\right)^{p_{2}-1}, \frac{1}{u_{20}}\left(\frac{\delta_{2}}{\sigma_{2}}\right)^{p_{2}-1}\right)$, and $v \in\left(0, \frac{1}{u_{30}}\left(\frac{\delta_{3}}{\sigma_{3}}\right)^{p_{3}-1}\right)$, system (1)-(2) has at least one positive solution.

(3) If $0<u_{10}, f_{\infty}, u_{20}, g_{\infty}, u_{30}, h_{\infty}<\infty,\left(\sigma_{3}\right)^{p_{3}-1} u_{30}<\left(\delta_{3} m^{2} \rho_{3}\right)^{p_{3}-1} h_{\infty}$, then for each $\lambda \in\left(0, \frac{1}{u_{10}}\left(\frac{\delta_{1}}{\sigma_{1}}\right)^{p_{1}-1}\right), \mu \in\left(0, \frac{1}{u_{20}}\left(\frac{\delta_{3}}{\sigma_{3}}\right)^{p_{2}-1}\right)$, and $v \in\left(\frac{1}{h_{\infty}}\left(\frac{1}{m^{2} \rho_{3}}\right)^{p_{3}-1}, \frac{1}{u_{30}}\left(\frac{\delta_{3}}{\sigma_{3}}\right)^{p_{3}-1}\right)$, system (1)-(2) has at least one positive solution.

Similarly, we can also obtain the following theorem that is in some way of duality of Corollary 3.5.

Corollary 3.6 Assume that conditions (A1)-(A4) are satisfied, $\xi_{1}, \xi_{2}, \xi_{3}>0$ with $\xi_{1}+\xi_{2}+$ $\xi_{3}=1$, then we have the following results:

(1) If $0<u_{1 \infty}, f_{0}, u_{2 \infty}, g_{0}, u_{3 \infty}, h_{0}<\infty,\left(\sigma_{1}\right)^{p_{1}-1} u_{1 \infty}<\left(\xi_{1} m^{2} \rho_{1}\right)^{p_{1}-1} f_{0}$, then for each $\lambda \in\left(\frac{1}{f_{0}}\left(\frac{1}{m^{2} \rho_{1}}\right)^{p_{1}-1}, \frac{1}{u_{1 \infty}}\left(\frac{\xi_{1}}{\sigma_{1}}\right)^{p_{1}-1}\right), \mu \in\left(0, \frac{1}{u_{2 \infty}}\left(\frac{\xi_{2}}{\sigma_{2}}\right)^{p_{2}-1}\right)$, and $v \in\left(0, \frac{1}{u_{3 \infty}}\left(\frac{\xi_{3}}{\sigma_{3}}\right)^{p_{3}-1}\right)$, system (1)-(2) has at least one positive solution.

(2) If $0<u_{1 \infty}, f_{0}, u_{2 \infty}, g_{0}, u_{3 \infty}, h_{0}<\infty,\left(\sigma_{2}\right)^{p_{2}-1} u_{2 \infty}<\left(\xi_{2} m^{2} \rho_{2}\right)^{p_{2}-1} g_{0}$, then for each $\lambda \in\left(0, \frac{1}{u_{1 \infty}}\left(\frac{\xi_{1}}{\sigma_{1}}\right)^{p_{1}-1}\right), \mu \in\left(\frac{1}{g_{0}}\left(\frac{1}{m^{2} \rho_{2}}\right)^{p_{2}-1}, \frac{1}{u_{2 \infty}}\left(\frac{\xi_{2}}{\sigma_{2}}\right)^{p_{2}-1}\right)$, and $v \in\left(0, \frac{1}{u_{3 \infty}}\left(\frac{\xi_{3}}{\sigma_{3}}\right)^{p_{3}-1}\right)$, system (1)-(2) has at least one positive solution.

(3) If $0<u_{1 \infty}, f_{0}, u_{2 \infty}, g_{0}, u_{3 \infty}, h_{0}<\infty,\left(\sigma_{3}\right)^{p_{3}-1} u_{3 \infty}<\left(\xi_{3} m^{2} \rho_{3}\right)^{p_{3}-1} h_{0}$, then for each $\lambda \in\left(0, \frac{1}{u_{1 \infty}}\left(\frac{\xi_{1}}{\sigma_{1}}\right)^{p_{1}-1}\right), \mu \in\left(0, \frac{1}{u_{2} \infty}\left(\frac{\xi_{2}}{\sigma_{2}}\right)^{p_{2}-1}\right)$, and $v \in\left(\frac{1}{h_{0}}\left(\frac{1}{m^{2} \rho_{3}}\right)^{p_{3}-1}, \frac{1}{u_{3} \infty}\left(\frac{\xi_{3}}{\sigma_{3}}\right)^{p_{3}-1}\right)$, system (1)-(2) has at least one positive solution.

Remark 3.1 Assume that (A1)-(A4) hold. If $u_{10}, u_{20}, u_{30}, u_{1 \infty}, u_{2 \infty}, u_{3 \infty}<\infty$ then there exist positive constants $\lambda_{0}, \mu_{0}$, and $v_{0}$ such that, for every $\lambda \in\left(0, \lambda_{0}\right), \mu \in\left(0, \mu_{0}\right)$, and $v \in$ $\left(0, v_{0}\right)$, the boundary value problem $(1)-(2)$ has no positive solution.

Remark 3.2 Assume that (A1)-(A4) hold.

(i) If $f_{0}, f_{\infty}>0$, then there exists a positive constant $\tilde{\lambda}_{0}$ such that, for every $\lambda>\tilde{\lambda}_{0}$, $\mu>0$, and $v>0$, the boundary value problem (1)-(2) has no positive solution.

(ii) If $g_{0}, g_{\infty}>0$, then there exists a positive constant $\tilde{\mu}_{0}$ such that, for every $\mu>\tilde{\mu}_{0}$, $\lambda>0$, and $v>0$, the boundary value problem (1)-(2) has no positive solution.

(iii) If $h_{0}, h_{\infty}>0$, then there exists a positive constant $\tilde{v}_{0}$ such that, for every $v>\tilde{v}_{0}$, $\lambda>0$, and $\mu>0$, the boundary value problem (1)-(2) has no positive solution.

Remark 3.3 Assume that (A1)-(A4) hold. If $f_{0}, f_{\infty}, g_{0}, g_{\infty}, h_{0}, h_{\infty}>0$, then there exist positive constants $\tilde{\tilde{\lambda}}_{0}, \tilde{\tilde{\mu}}_{0}$, and $\tilde{\tilde{v}}_{0}$ such that, for every $\lambda>\tilde{\tilde{\lambda}}_{0}, \mu>\tilde{\tilde{\mu}}_{0}$, and $v>\tilde{\tilde{v}}_{0}$, the boundary value problem (1)-(2) has no positive solution.

\section{Example}

Let us consider an example to illustrate the above results.

Let $\alpha_{1}=\alpha_{2}=\alpha_{3}=5 / 2, \beta_{1}=\beta_{2}=\beta_{3}=3 / 2, \xi_{1}=\xi_{2}=\xi_{3}=4, \eta_{1}=\eta_{2}=\eta_{3}=8, \gamma_{1}=\gamma_{2}=\gamma_{3}=$ $3 / 2, v_{1}=v_{2}=v_{3}=1 / 2, \delta=3 / 2, p_{1}=p_{2}=p_{3}=2, q_{1}=q_{2}=q_{3}=2, \phi_{p_{1}}(s)=s, \phi_{q_{1}}(s)=s$. 
We consider the system of Hadamard fractional differential equation

$$
\begin{cases}{ }^{H} D_{1^{+}}^{3 / 2}\left(\phi_{p_{1}}\left({ }^{H} D_{1^{+}}^{5 / 2} x(t)\right)\right)=\lambda f(t, x(t), y(t), z(t)), & t \in(1, e), \\ { }^{H} D_{1^{+}}^{3 / 2}\left(\phi_{p_{2}}\left({ }^{H} D_{1^{+}}^{5 / 2} y(t)\right)\right)=\mu g(t, x(t), y(t), z(t)), & t \in(1, e), \\ { }^{H} D_{1^{+}}^{3 / 2}\left(\phi_{p_{3}}\left({ }^{H} D_{1^{+}}^{5 / 2} z(t)\right)\right)=v h(t, x(t), y(t), z(t)), & t \in(1, e),\end{cases}
$$

with the three-point boundary conditions

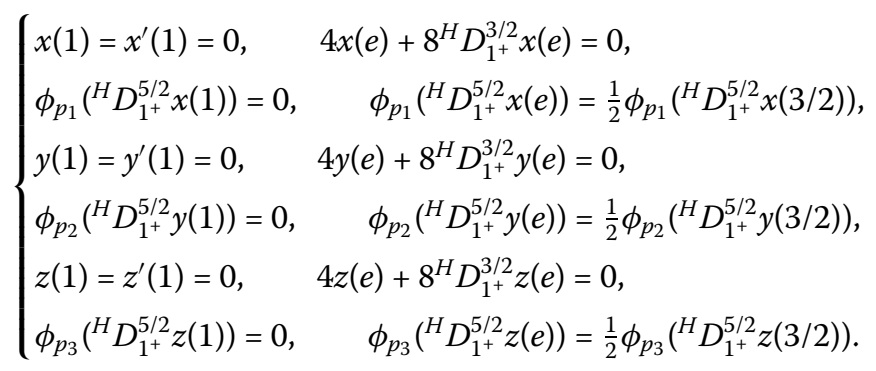

Here, $f(t, x, y, z)=(1-\ln t)^{1 / 2}\left(1+\sin ^{2}(x+y+z)\right), g(t, x, y, z)=\ln ^{1 / 2} t \sin (x+y+z)$, and $h(t, x, y, z)=(1+\ln t)^{2}\left(e^{(x+y+z)^{3}}+\sin y\right)$ for all $t \in(1, e), x, y, z \geq 0$. Then we obtain $\Delta_{1}=\Delta_{2}=$ $\Delta_{3} \approx 14.635>0, m=0.125$, and assumptions (A1) and (A2) are satisfied. In addition, we deduce that

$$
\begin{aligned}
& G_{1}(t, s)= \begin{cases}G_{11}(t, s), & 1 \leq t \leq s \leq e, \\
G_{12}(t, s), & 1 \leq s \leq t \leq e\end{cases} \\
& G_{11}(t, s)=\frac{1}{\Delta_{1}}\left[8(1-\ln s)^{-3 / 2}+\frac{4}{\Gamma(5 / 2)}\right](\ln t)^{3 / 2}(1-\ln s)^{3 / 2}, \\
& G_{2}(t, s)=\frac{1}{\Delta_{1}}\left[8(1-\ln s)^{-3 / 2}+\frac{4}{\Gamma 5 / 2}\right](\ln t)^{3 / 2}(1-\ln s)^{3 / 2}-\frac{1}{\Gamma(5 / 2)}\left(\ln \frac{t}{s}\right)^{3 / 2},
\end{aligned}
$$

and

$$
K_{1}(t, s)=K_{11}(t, s)+\frac{1 / 2(\ln t)^{1 / 2}}{1-1 / 2(\ln (3 / 2))^{1 / 2}} K_{11}(3 / 2, s)
$$

where

$$
\begin{aligned}
& K_{11}(t, s)=\frac{1}{\Gamma(3 / 2)} \begin{cases}(\ln t)^{1 / 2}(1-\ln s)^{1 / 2}, & 1 \leq t \leq s \leq e, \\
(\ln t)^{1 / 2}(1-\ln s)^{1 / 2}-\left(\ln \frac{t}{s}\right)^{1 / 2}, & 1 \leq s \leq t \leq e,\end{cases} \\
& \int_{1}^{e} G_{1}(e, s) \frac{d s}{s}= \int_{1}^{e}\left[\frac{1}{14.635}\left[8(1-\ln s)^{-3 / 2}+\frac{4 \Gamma(1)}{\Gamma(5 / 2)}\right](1-\ln s)^{3 / 2}\right. \\
&\left.-\frac{1}{\Gamma(5 / 2)}(1-\ln s)^{3 / 2}\right] \frac{d s}{s} \\
&= 0.5466+\frac{16(0.06833)}{5 \sqrt{\pi}} \int_{1}^{e}(1-\ln s)^{3 / 2} \frac{d s}{s}-\frac{4}{3 \sqrt{\pi}} \int_{1}^{e}(1-\ln s)^{3 / 2} \frac{d s}{s} \\
&= 0.5466+\frac{32}{25 \sqrt{\pi}}(0.06833)-\frac{8}{15 \sqrt{\pi}}
\end{aligned}
$$




$$
\begin{aligned}
& =0.5466+0.04935-0.3009=0.29505 \text {, } \\
& \int_{1}^{e} K_{11}(\tau, \tau) \frac{d \tau}{\tau}=\int_{1}^{e} \frac{(\ln \tau)^{\beta_{1}-1}(1-\ln \tau)^{\beta_{1}-1}}{\Gamma\left(\beta_{1}\right)} \frac{d \tau}{\tau} \\
& =\frac{1}{\Gamma\left(\beta_{1}\right)} \int_{0}^{1} t^{\beta_{1}-1}(1-t)^{\beta_{1}-1} d t \\
& =\frac{\Gamma\left(\beta_{1}\right)}{\Gamma\left(2 \beta_{1}\right)}=\frac{\Gamma(3 / 2)}{\Gamma(3)}=\frac{\sqrt{\pi}}{4}, \\
& \sigma_{1}=\int_{1}^{e} G_{1}(e, s) \phi_{q_{1}}\left(\int_{1}^{e} l_{1}(\tau) \kappa_{1}(\tau) \frac{d \tau}{\tau}\right) \frac{d s}{s} \\
& =(0.29505)\left(\frac{\sqrt{\pi}}{4}+\frac{0.1825}{\sqrt{\pi}(0.7973)}\right) \approx 0.1689, \\
& \rho_{1}=\int_{s \in I} G_{1}(e, s) \phi_{q_{1}}\left(\int_{s \in I} \varsigma(\tau) K_{11}(\tau, \tau) \frac{d \tau}{\tau}\right) \frac{d s}{s} \approx 0.005876 .
\end{aligned}
$$

We choose $r_{1}=1, M=3, K=8000, \varrho_{1}=1 / 2, \varrho_{2}=1 / 3, \varrho_{3}=1 / 6$, then all the conditions in Theorem 3.3 are satisfied. Therefore, for any

$$
\begin{aligned}
& \lambda \in[0.1701837985,0.9867771857], \quad \mu \in(0,0.6578514571], \\
& \nu \in(0,0.3289257286] \quad \text { or } \\
& \lambda \in(0,0.9867771857], \quad \mu \in[0.1701837985,0.6578514571], \\
& \nu \in(0,0.3289257286] \quad \text { or } \\
& \lambda \in[0,0.9867771857], \quad \mu \in(0,0.6578514571], \\
& \nu \in[0.1701837985,0.3289257286],
\end{aligned}
$$

system (15)-(16) has at least two positive solutions $\left(x_{1}(t), y_{1}(t), z_{1}(t)\right),\left(x_{2}(t), y_{2}(t), z_{2}(t)\right)$ with $0<\left\|\left(x_{1}(t), y_{1}(t), z_{1}(t)\right)\right\|<1<\left\|\left(x_{2}(t), y_{2}(t), z_{2}(t)\right)\right\|$.

We choose $d_{1}=1, d_{2}=10, d_{3}=200, K=4, M=7000, \varsigma_{1}=1 / 3, \varsigma_{2}=1 / 6, \varsigma_{3}=1 / 2$, then all the conditions in Theorem 3.4 are satisfied. Therefore, for any

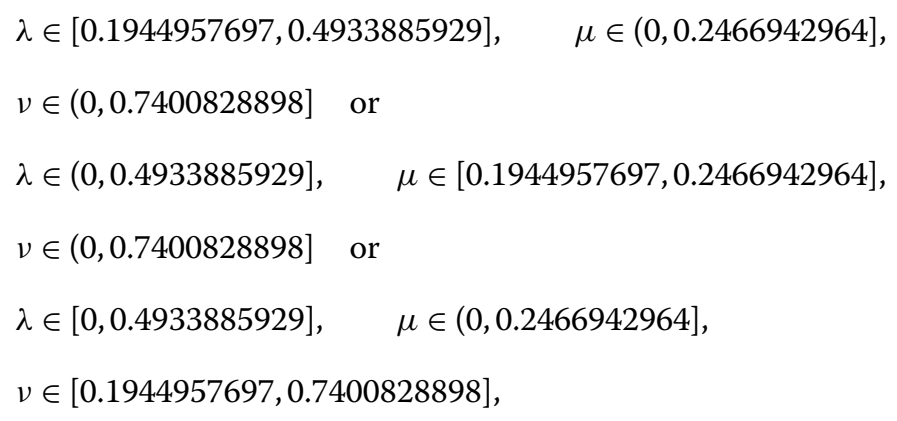

system (15)-(16) has at least two positive solutions $\left(x_{1}^{\star}(t), y_{1}^{\star}(t), z_{1}^{\star}(t)\right),\left(x_{2}^{\star}(t), y_{2}^{\star}(t), z_{2}^{\star}(t)\right)$ with $d_{1} \leq\left\|\left(x_{1}^{\star}(t), y_{1}^{\star}(t), z_{1}^{\star}(t)\right)\right\|<d_{2}<\left\|\left(x_{2}^{\star}(t), y_{2}^{\star}(t), z_{2}^{\star}(t)\right)\right\| \leq d_{3}$. 


\section{Conclusions}

By using Krasnosel'skii's fixed point theorem and under suitable conditions, we have presented the existence of multiplicity results of positive solutions to the system of three Hadamard fractional differential equations with $p$-Laplacian operator. We have also derived three explicit eigenvalue intervals of $\lambda, \mu$, and $v$ for the existence of multiple positive solutions. Finally, we have given an example to demonstrate our result.

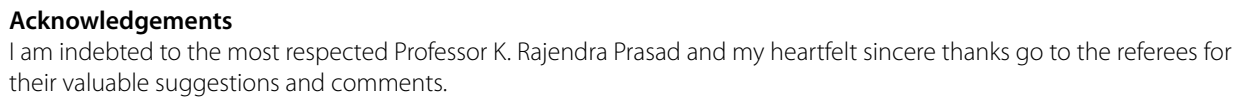

Availability of data and materials

Not applicable.

Competing interests

The authors declare that they have no competing interests.

Authors' contributions

All authors contributed equally and significantly in writing this article. All authors read and approved the final manuscript.

\section{Authors' information}

Manoj Singh, Department of Mathematics, Faculty of Science, Jazan University, Jazan, Saudi Arabia. email: msingh@jazanu.edu.sa; M. Zico Meetei: Department of Mathematics, Faculty of Science, Jazan University, Jazan, Saudi Arabia. email: mmeetei@jazanu.edu.sa.

\section{Publisher's Note}

Springer Nature remains neutral with regard to jurisdictional claims in published maps and institutional affiliations.

Received: 12 October 2019 Accepted: 18 February 2020 Published online: 02 March 2020

\section{References}

1. Torvik, P., Bagley, R.: On the appearance of the fractional derivative in the behavior of real materials. J. Appl. Mech. 51, 294-298 (1984)

2. Bai, J., Feng, X.: Fractional-order anisotropic diffusion for image denoising. IEEE Trans. Image Process. 16, 2492-2502 (2007)

3. Caponetto, R., Dongola, G., Fortuna, L., Petráš, I.: Fractional Order Systems: Modeling and Control Applications. World Scientific, River Edge (2010)

4. Chern, J.: Finite element modeling of viscoelastic materials on the theory of fractional calculus. Ph.D. thesis, Pennsylvania State University (1993)

5. Freed, A., Diethelm, K., Luchko, Y.: Fractional-order viscoelasticity (FOV): constitutive development using the fractional calculus (first annual report). Technical Memorandum 2002-211914, NASA Glenn Research Center, Cleveland (2002)

6. Freed, A., Diethelm, K.: Fractional calculus in biomechanics: a 3D viscoelastic model using regularized fractional-derivative kernels with application to the human calcaneal fat pad. Biomech. Model. Mechanobiol. 5, 203-215 (2006)

7. Magin, R.: Fractional Calculus in Bioengineering. Begell House, Redding (2006)

8. Kiryakova, $V_{\text {:: }}$ The special functions of fractional calculus as generalized fractional calculus operators of some basic functions. Comput. Math. Appl. 59, 1128-1141 (2010)

9. Kilbas, A.A., Srivastava, H.M., Trujillo, J.J.: Theory and Applications of Fractional Differential Equations. Elsevier, Amsterdam (2006)

10. Podlubny, I.: Fractional Differential Equations. Academic Press, New York (1999)

11. Miller, K.S., Ross, B.: An Introduction to the Fractional Calculus and Fractional Differential Equations. Wiley, New York (1993)

12. Sabatier, J., Agrawal, O.P., Machado, J.T.: Advances in Fractional Calculus, Theoretical Developments and Applications in Physics and Enggineering. Springer, Dordrecht (2007)

13. Papageorglou, N.S., Radulescu, V.D., Repovs, D.D.: Nonlinear Analysis-Theory and Methods. Springer Monographs in Mathematics. Springer, Cham (2019)

14. Leibenson, L.S.: General problem of the movement of a compressible fluid in a porous medium. Izv. Akad. Nauk SSSR Ser. Geogr. Geofiz. 9, 7-10 (1945)

15. Bachar, l., Maagli, H., Radulescu, V.D.: Fractional Navier boundary value problems. Bound. Value Probl. 2016, 79 (2016)

16. Molica Bisci, G., Radulescu, V.D.: Ground state solutions of scalar field fractional Schrödinger equations. Calc. Var. Partial Differ. Equ. 54(3), 2985-3008 (2015) 
17. Chidouh, A., Torres, D.: Existence of positive solutions to a discrete fractional boundary value problem and corresponding Lyapunov-type inequalities. Opusc. Math. 38(1), 31-40 (2018)

18. Fečkan, M., Wang, J.R.: Periodic impulsive fractional differential equations. Adv. Nonlinear Anal. 8(1), $482-496$ (2019)

19. Binlin, Z., Radulescu, V.D., Wang, L.: Existence results for Kirchhoff-type superlinear problems involving the fractional Laplacian. Proc. R. Soc. Edinb., Sect. A 149(4), 1061-1081 (2019)

20. Musina, R., Nazarov, A.: A note on truncations in fractional Sobolev spaces. Bull. Math. Sci. 9(1), 1950001 (2019)

21. Takahashi, F.: Critical and subcritical fractional Trudinger-Moser-type inequalities on $\mathbb{R}$. Adv. Nonlinear Anal. 8(1), 868-884 (2019)

22. Luca, R.: Positive solutions for a system of Riemann-Liouville fractional differential equations with multi-point fractional boundary conditions. Bound. Value Probl. 2017, 102 (2017)

23. Chai, G.: Positive solutions for boundary value problem of fractional differential equation with $p$-Laplacian operator. Bound. Value Probl. 2012, 18 (2012)

24. $\mathrm{Xu}, \mathrm{J}, \mathrm{O}$ 'Regan, D.: Positive solutions for a fractional $p$-Laplacian boundary value problem. Filomat 31(6), 1549-1558 (2017)

25. Tian, Y., Wei, Y., Sun, S.: Multiplicity for fractional differential equations with $p$-Laplacian. Bound. Value Probl. 2018, 127 (2018)

26. Tian, Y., Bai, Z., Sun, S.: Positive solutions for a boundary value problem of fractional differential equation with p-Laplacian operator. Adv. Differ. Equ. 2019, 349 (2019)

27. Yan, F., Zuo, M., Hao, X.: Positive solution for a fractional singular boundary value problem with $p$-Laplacian operator. Bound. Value Probl. 2018, 51 (2018)

28. Rao, S.N.: Multiple positive solutions for a system of Riemann-Liouville fractional order two-point boundary value problems. Panam. Math. J. 25(1), 66-81 (2015)

29. Prasad, K.R., Krushna, B.M.B.: Multiple positive solutions for a coupled system of $p$-Laplacian fractional order two-point boundary value problems. Int. J. Differ. Equ. 2014, Article ID 485647 (2014)

30. Rao, S.N.: Multiplicity of positive solutions for fractional differential equation with $p$-Laplacian boundary value problems. Int. J. Differ. Equ. 2016, Article ID 6906049 (2016)

31. Dong, X., Bai, Z., Zhang, S.: Positive solutions to boundary value problems of $p$-Laplacian with fractional derivative. Bound. Value Probl. 2017, 5 (2017)

32. He, J., Song, X.: The uniqueness of solution for a class of fractional order nonlinear systems with $p$-Laplacian operator. Abstr. Appl. Anal. 2014, Article ID 921209 (2014)

33. Hao, X., Wang, H., Liu, L., Cui, Y.: Positive solutions for a system of nonlinear fractional nonlocal boundary value problems with parameters and p-Laplacian operator. Bound. Value Probl. 2017, 182 (2017)

34. Liu, Y., Xie, D., Bai, C., Yang, D.: Multiple positive solutions for a coupled system of fractional multi-point BVP with p-Laplacian operator. Adv. Differ. Equ. 2017, 168 (2017)

35. Rao, S.N.: Multiple positive solutions for coupled system of $p$-Laplacian fractional order three-point boundary value problems. Rocky Mt. J. Math. 49, 2 (2019)

36. Luca, R.: Positive solutions for a system of fractional differential equations with $p$-Laplacian operator and multi-point boundary conditions. Nonlinear Anal., Model. Control 23(5), 771-801 (2018)

37. Hadamard, J.: Essai sur letude des fonctions donnees par leur developpement de Taylor. J. Math. Pures Appl. 8, $101-186(1892)$

38. Wang, J., Zhang, Y.: On the concept and existence of solutions for fractional impulsive systems with Hadamard derivatives. Appl. Math. Lett. 39, 85-90 (2015)

39. Huang, H., Liu, W.: Positive solutions for a class of nonlinear Hadamard fractional differential equations with a parameter. Adv. Differ. Equ. 2018, 96 (2018)

40. Zhai, C., Wang, W., Li, H.: A uniqueness method to a new Hadamard fractional differential system with four-point boundary conditions. J. Inequal. Appl. 2018, 207 (2018)

41. Yang, W.: Positive solutions for singular coupled integral boundary value problems of nonlinear Hadamard fractional differential equations. J. Nonlinear Sci. Appl. 8, 110-129 (2015)

42. Yang, W.: Positive solutions for singular Hadamard fractional differential system with four-point coupled boundary conditions. J. Appl. Math. Comput. 49, 357-381 (2015)

43. Li, Y.L., Lin, S.Y.: Positive solution for the nonlinear Hadamard type fractional differential equation with $p$-Laplacian. J. Funct. Spaces Appl. 2013, Article ID 951643 (2013)

44. Wang, G., Wang, T.: On a nonlinear Hadamard type fractional differential equation with $p$-Laplacian operator and strip condition. J. Nonlinear Sci. Appl. 9, 5073-5081 (2016)

45. Zhang, K., Wang, J., Ma, W.: Solutions for integral boundary value problems of nonlinear Hadamard fractional differential equations. J. Funct. Spaces 2018, Article ID 2193234 (2018)

46. Li, S., Zhai, C.: Positive solutions for a new class of Hadamard fractional differential equations on infinite intervals. J. Inequal. Appl. 2019, 50 (2019)

47. Zhang, W., Liu, W.: Existence of solutions for several higher-order Hadamard-type fractional differential equations with integral boundary conditions on infinite interval. Bound. Value Probl. 2018, 134 (2018)

48. Ahmad, B., Ntouyas, S.K.: A fully Hadamard type integral boundary value problem of a coupled system of fractional differential equations. Fract. Calc. Appl. Anal. 17, 348-360 (2014)

49. Aljoudi, S., Ahmad, B., Nieto, J.J., Alsaedi, A.: On coupled Hadamard type sequential fractional differential equations with variable coefficients and nonlocal integral boundary conditions. Filomat 31, 6041-6049 (2017)

50. Zhang, W., Liu, W.: Existence, uniqueness, and multiplicity results on positive solutions for a class of Hadamard-type fractional boundary value problem on an infinite interval. Math. Methods Appl. Sci. (2019). https://doi.org/10.1002/mma.6038

51. Jiang, J., O'Regan, J., Xu, J., Fu, Z.: Positive solutions for a system of nonlinear Hadamard fractional differential equations involving coupled integral boundary conditions. J. Inequal. Appl. 2019, 204 (2019)

52. Zhai, C., Wang, W.: Solutions for a system of Hadamard fractional differential equations with integral conditions. Numer. Funct. Anal. Optim. 41(7), 1-21 (2019) 
53. Asawasamrit, S., Ntouyas, S.K., Tariboon, J., Nithiarayaphaks, W.: Coupled systems of sequential Caputo and Hadamard fractional differential equations with coupled separated boundary conditions. Symmetry 10, 701 (2018). https://doi.org/10.3390/sym10120701

54. Alesemi, M.: Solvability for a class of nonlinear Hadamard fractional differential equations with parameters. Bound. Value Probl. 2019, 101 (2019)

55. Guo, D.J., Lakshmikantham, V.: Nonlinear Problems in Abstract Cones. Academic Press, Boston (1988)

56. Krasnosel'skii, M.A.: Positive Solutions of Operator Equations. Noordhoff, Groningen (1964)

Submit your manuscript to a SpringerOpen ${ }^{\circ}$ journal and benefit from:

- Convenient online submission

$\checkmark$ Rigorous peer review

- Open access: articles freely available online

- High visibility within the field

- Retaining the copyright to your article

Submit your next manuscript at $\gg$ springeropen.com 